\title{
Lightwave-driven quasiparticle collisions on a subcycle timescale
}

\author{
F. Langer ${ }^{1}$, M. Hohenleutner ${ }^{1}$, C. P. Schmid ${ }^{1}$, C. Poellmann ${ }^{1}$, P. Nagler ${ }^{1}$, T. Korn ${ }^{1}$, C. Schüller ${ }^{1}$, M. S. Sherwin ${ }^{2}$, U. Huttner ${ }^{3}$,
} J. T. Steiner ${ }^{3}$, S. W. Koch ${ }^{3}$, M. Kira ${ }^{3} \&$ R. Huber ${ }^{1}$

Ever since Ernest Rutherford scattered $\alpha$-particles from gold foils ${ }^{1}$, collision experiments have revealed insights into atoms, nuclei and elementary particles ${ }^{2}$. In solids, many-body correlations lead to characteristic resonances ${ }^{3}$ - called quasiparticles-such as excitons, dropletons ${ }^{4}$, polarons and Cooper pairs. The structure and dynamics of quasiparticles are important because they define macroscopic phenomena such as Mott insulating states, spontaneous spin- and charge-order, and high-temperature superconductivity ${ }^{5}$. However, the extremely short lifetimes of these entities ${ }^{6}$ make practical implementations of a suitable collider challenging. Here we exploit lightwave-driven charge transport ${ }^{7-24}$, the foundation of attosecond science ${ }^{9-13}$, to explore ultrafast quasiparticle collisions directly in the time domain: a femtosecond optical pulse creates excitonic electron-hole pairs in the layered dichalcogenide tungsten diselenide while a strong terahertz field accelerates and collides the electrons with the holes. The underlying dynamics of the wave packets, including collision, pair annihilation, quantum interference and dephasing, are detected as light emission in high-order spectral sidebands ${ }^{17-19}$ of the optical excitation. A full quantum theory explains our observations microscopically. This approach enables collision experiments with various complex quasiparticles and suggests a promising new way of generating sub-femtosecond pulses.

Following the principle of high-energy accelerators, their solid-state counterparts should prepare, accelerate and collide quasiparticles while detecting the outcomes. This can be realized only if all steps are accomplished faster than the ultrashort lifetime of the quasiparticle ${ }^{6}$. Preparing the quantum states of quasiparticles by femtosecond lasers is already well established ${ }^{25}$. Ultrafast acceleration, in turn, may harness the latest breakthroughs of strong-field control: the carrier wave of an intense laser pulse has been applied to ionize atoms or molecules and recollide their fragments when the sign of the light-field changes ${ }^{10}$. The excess energy of the collision is released as high-order harmonics of the laser frequency, which may emerge in attosecond bursts ${ }^{9}$, carrying key structural and dynamical information about the participants ${ }^{11,12}$. Recently, this idea of subcycle control has been extended to solids, in which lightwaves have driven dynamical Bloch oscillations ${ }^{20,21}$, interband quantum interference ${ }^{24}$ and high-order harmonic generation $^{20-24}$ (HHG). However, the microscopic mechanisms are complex because the lightwave drives an inseparable combination of interband excitation ${ }^{21,24,26}$ (quasiparticle preparation) and intraband acceleration $^{20-24,26}$. The recent observation of high-order sideband generation (HSG) in gallium arsenide quantum wells ${ }^{18}$ has suggested a way to disentangle these steps. Following optical preparation of coherent excitons $^{27}$, a strong terahertz $(\mathrm{THz})$ field of frequency $\nu_{\mathrm{THz}}$ modulates the interband resonance such that spectral sidebands at even multiples of $\nu_{\mathrm{THz}}$ emerge $\mathrm{e}^{17-19}$. This observation has been modelled by THz-driven electron-hole collisions, similarly to the three-step model of HHG in atomic gases ${ }^{7,8}$. Yet, the potential of HSG for real-time quasiparticle collisions has remained untapped.
Here, we introduce a subcycle quasiparticle collider. A femtosecond optical pulse prepares coherent excitons in tungsten diselenide ( $\left.\mathrm{WSe}_{2}\right)$, at a well-defined phase of a $\mathrm{THz}$ driving field $\left(E_{\mathrm{THz}}\right)$. This phase defines how $E_{\mathrm{THz}}$ accelerates and collides electrons and holes, thereby yielding photon emission by pair annihilation, which is detected as high-order sidebands. We reveal the subcycle quantum dynamics of quasiparticle collisions, model the microscopic process by a full quantum theory and use the data to extract key information on the colliding species. Finally, we compare the process to HHG in the same material.

As a member of the class of transition metal dichalcogenides, $\mathrm{WSe}_{2}$ features strongly bound Wannier excitons ${ }^{27,28}$ and unique spin-valley coupling $^{29}$. Even in its bulk form, $\mathrm{WSe}_{2}$ has been reported to exhibit an exciton binding energy of the order of $0.1 \mathrm{eV}$ at its direct bandgap ${ }^{30}$, which implies that these quasiparticles are stable at room temperature. In a first experiment, a 100-fs excitation pulse (red waveform in Fig. 1a) centred at a photon energy of $1.621 \mathrm{eV}$ (centre frequency, $392 \mathrm{THz}$ ) resonantly prepares coherent excitons at the direct band gap of $\mathrm{WSe}_{2}$ (thickness, $60 \mathrm{~nm}$ ) located at the $\mathrm{K}$ and $\mathrm{K}^{\prime}$ points in momentum space. A co-propagating intense $\mathrm{THz}$ pulse (blue waveform in Fig. 1a, b; centre photon energy, $h \nu_{\mathrm{THz}}=95 \mathrm{meV}$ ( $h$ is the Planck constant); centre frequency, $\nu_{\mathrm{THz}}=23 \mathrm{THz}$; peak electric field in air, $E_{\text {peak }}=17 \mathrm{MV} \mathrm{cm}^{-1}$; see Methods section 'Experimental set-up') modulates the excitonic polarization and gives rise to $\mathrm{HSG}^{18,19}$. The resulting intensity spectrum $I_{\mathrm{HSG}}$ includes sidebands of positive and negative orders $n$, spaced at $2 \nu_{\mathrm{THz}}$ (Fig. 1b). Spectral components up to $n=22$ are clearly visible, while the intensities of negative orders drop much faster with decreasing frequencies. The new frequency components feature the same polarization as the interband excitation pulse (Extended Data Fig. 1). In contrast to perturbative scaling $\left(I_{\mathrm{HSG}} \propto E_{\text {peak }}^{2 n}\right)$, the sideband intensity grows linearly with $E_{\text {peak }}$ for intermediate peak fields, depending on the sideband order, and even saturates at the highest field strengths (Fig. 1c, see also Extended Data Fig. 2 for the influence of the interband excitation fluence). Finally, $I_{\mathrm{HSG}}$ peaks if the interband excitation is resonant with the excitonic absorption peak (Fig. 1d), underpinning its excitonic origin. Apart from the broadening of the sidebands due to the ultrashort duration of $E_{\mathrm{THz}}$, these findings agree well with previous time-integrated studies ${ }^{18}$.

To directly resolve the underlying subcycle quantum dynamics, we now prepare the coherent excitons within $10 \mathrm{fs}$ - much faster than a single oscillation period $T=\nu_{\mathrm{THz}}^{-1}$ of the $\mathrm{THz}$ wave. Figure $2 \mathrm{a}$ depicts the spectral shape of the observed sideband intensity $I_{\mathrm{HSG}}$ as a function of the delay between the excitation pulse and the peak of the $\mathrm{THz}$ driving field, $t_{\mathrm{ex}}$. Electro-optic detection allows us to determine the complete waveform $E_{\mathrm{THz}}$ on the same absolute timescale (Fig. 2a, b, blue curve). The spectrogram in Fig. 2a follows a nearly Gaussian temporal envelope, resembling the $\mathrm{THz}$ envelope. Interestingly, $I_{\mathrm{HSG}}$ is strongly modulated along the time axis with a period of $T / 2$; that is, there is a subcycle criterion for 'good' and 'bad' preparation times $t_{\mathrm{ex}}$. 


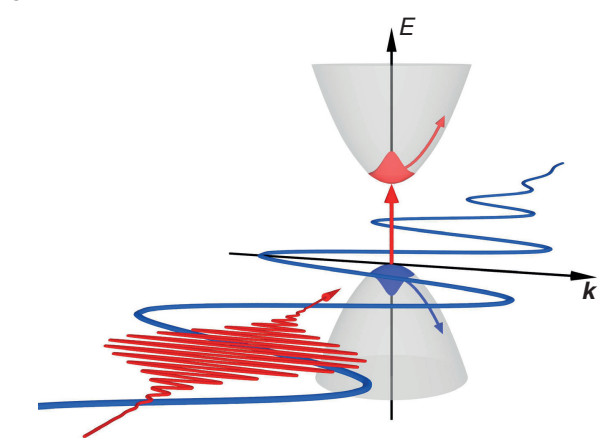

c

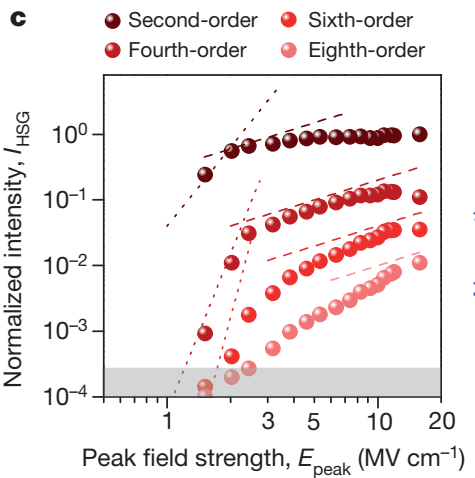

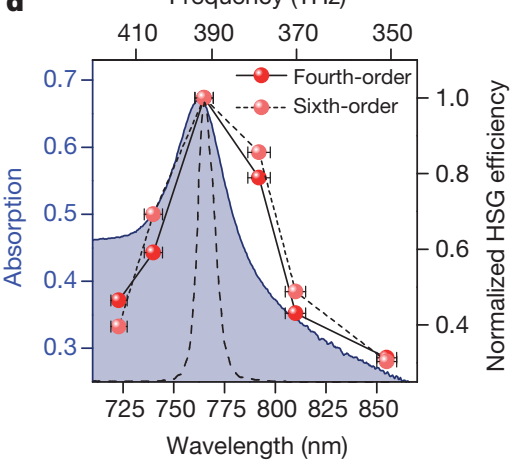

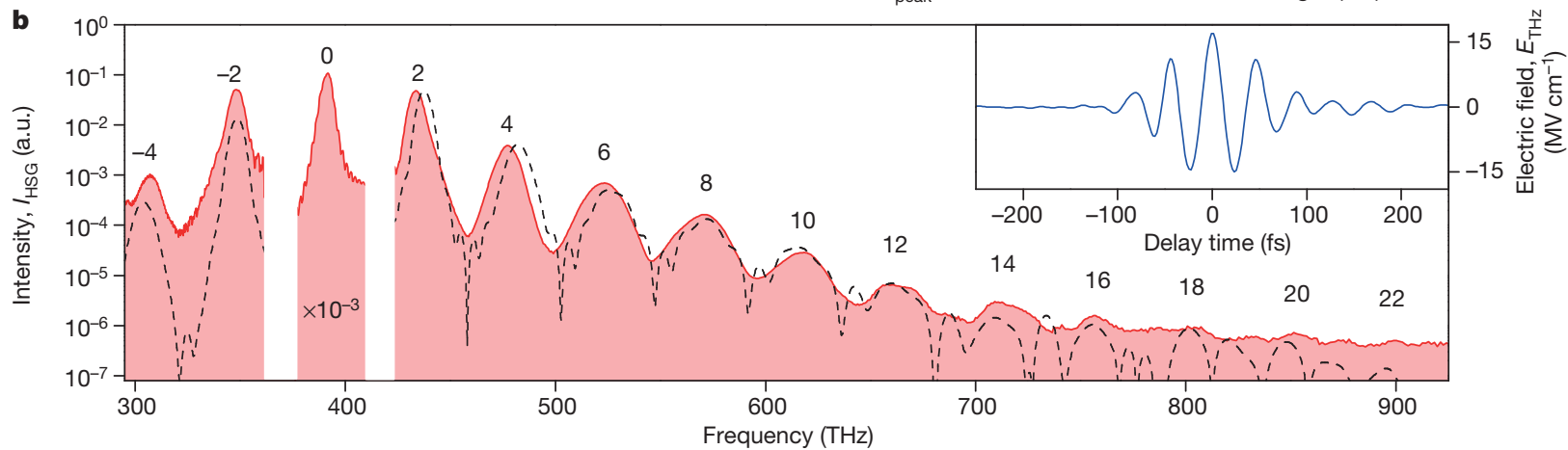

Figure $1 \mid$ High-order sideband generation in tungsten diselenide. a, Schematic of the experiment in reciprocal space ( $E$, energy; $\boldsymbol{k}$, wave vector): an interband excitation pulse (red waveform) creates an excitonic polarization in $\mathrm{WSe}_{2}$ (red, vertical arrow), while a strong multi-THz field (blue waveform) simultaneously accelerates the wave packets of the electron and hole (curved arrows) within their respective bands (grey shaded parabolas). b, Measured intensity spectrum (red) of high-order sidebands from $\mathrm{WSe}_{2}$ (thickness, $60 \mathrm{~nm}$; sample at room temperature) driven by a phase-locked $\mathrm{THz}$ transient featuring a centre frequency of $23 \mathrm{THz}$ and an external peak field strength of $E_{\mathrm{THz}}=17 \mathrm{MV} \mathrm{cm}^{-1}$ (see inset; Keldysh parameter $\gamma=0.08<1$ ) under resonant optical excitation at a frequency of $392 \mathrm{THz}$ (labelled by '0', 0th order has been multiplied by a

For a quantitative timing analysis, Fig. $2 \mathrm{~b}$ compares $E_{\mathrm{THz}}$ (blue curve) with the spectrally integrated intensity $I_{\mathrm{HSG}}$ (red shaded curve). The strongest THz half-cycle occurs at a delay $\delta_{\text {global }} \approx T$ with respect to the most-intense sideband peak. This retardation rules out instantaneous optical nonlinearities as the microscopic origin of HSG. A detailed comparison of $E_{\mathrm{THz}}$ and $I_{\mathrm{HSG}}$ also reveals a subcycle delay $\delta_{\mathrm{sc}}$ of the observed sideband bursts relative to the THz crests (see inset in Fig. $2 \mathrm{~b}$ ). For subsequent half-cycles of the driving field, $\delta_{\mathrm{sc}}$ initially increases, reaches a maximum value of approximately $T / 8$ at the centre of the THz pulse and slightly decreases afterwards (Fig. 2d).

To link these signatures of $I_{\mathrm{HSG}}$ with the microscopic quasiparticle motion, we analyse the experiment using a full quantum mechanical model based on the semiconductor Bloch equations (see Methods section 'Microscopic model of HSG'). For a 100 -fs preparation pulse, the experimentally observed HSG spectrum is well reproduced by our calculations (Fig. 1b, black dashed curve), showing a similar modulation and intensity roll-off on the high- and low-frequency sides. Using a 10 -fs preparation pulse, the calculations quantitatively reproduce the temporal characteristics of $I_{\mathrm{HSG}}$ (Fig. 2c), including even delicate features such as $\delta_{\text {sc }}$ (Fig. 2d).

Figure 3 visualizes the underlying microscopic quasiparticle dynamics for two characteristic preparation times $t_{\mathrm{ex}}$, corresponding to mini$\operatorname{mum}\left(t_{\mathrm{ex}}=-4\right.$ fs, Fig. $\left.3 \mathrm{c}, \mathrm{e}, \mathrm{g}\right)$ and maximum $\left(t_{\mathrm{ex}}=7 \mathrm{fs}\right.$, Fig. 3d, f, h) HSG. The two quasiparticle-collider sequences are schematically illustrated by Feynman diagrams in Fig. 3a, b. For $t_{\mathrm{ex}}=-4 \mathrm{fs}$, the coherent excitons are prepared shortly before a THz field crest (see vertical dashed lines in Figs $2 \mathrm{c}$ and $3 \mathrm{c}$ ). Immediately after optical preparation, the average momenta of the electron (Fig. 3e, solid curve) factor of $10^{-3}$; other numerals denote the order of sidebands). The black dashed curve shows the calculated intensity spectrum $I_{\mathrm{HSG}}$. a.u., arbitrary units. c, Recorded high-order sideband intensity $I_{\mathrm{HSG}}$ of orders $(n)$ two to eight as a function of driving peak field strength $E_{\text {peak }}$. Dotted lines follow a perturbative scaling law, $I_{\mathrm{HSG}} \propto E_{\text {peak }}^{2 n}$; dashed lines mark a linear scaling; the grey shaded area indicates the noise level. d, Measured generation efficiency of the fourth- and sixth-order sideband for different excitation wavelengths (red spheres). Error bars represent the bandwidth (full-width at half maximum) of the excitation spectra (excitation spectrum at $392 \mathrm{THz}$ shown as a dashed curve). The shaded area shows the measured exciton resonance in the absorption spectrum of the sample.

and the hole (dashed curve) are located in the vicinity of the $\mathrm{K}$ and $\mathrm{K}^{\prime}$ points of the Brillouin zone (defined as $k=0$ ). The strong THz field accelerates the distribution function of the electron $\left(f_{k}^{\mathrm{e}}\right.$; Fig. 3e, false-colour plot) and the hole ( $f_{k}^{\mathrm{h}}$; not shown) away from $k=0$, driving the electron and hole wave packets apart. A qualitatively different situation occurs for $t_{\mathrm{ex}}=7 \mathrm{fs}$ (Fig. $3 \mathrm{~d}, \mathrm{f}, \mathrm{h}$ ), for which the coherent excitons are prepared shortly before a zero crossing of the $\mathrm{THz}$ field (vertical dashed lines in Figs $2 \mathrm{c}$ and 3d). Now the mean momenta change sign; that is, the initially separating electron and hole are set on a recollision path (Fig. 3f).

Particle preparation of typical accelerators is spatially precise and collisions are monitored in real space. Our concept follows the relative motion of quasiparticles, and the preparation is precise in terms of the relative coordinates of the quasiparticles because the optical pulse creates them in a single, spatially correlated excitonic state described by a pair correlation function ${ }^{27}$ (see Methods section 'Coherent electronhole pair-correlation function'). Figure $3 \mathrm{~g}$, h displays its coherent part $g_{\mathrm{coh}}(r)$, which defines how electrons are coherently coupled to holes as a function of their relative distance $r$ (reciprocal space representation in Extended Data Fig. 3). In both panels, $g_{\text {coh }}(r)$ is maximum close to the peak of the optical pulse before it decays by ultrafast dephasing (dephasing time $T_{2}<T / 2$ ). Figure $3 c$, d shows the corresponding mean electron-hole separation $\langle r\rangle$. Suitable $t_{\mathrm{ex}}$ (Fig. 3d) allows the THz field (Fig. 3c, d, blue curve) to separate and collide the electron-hole pairs at high energy. Owing to this ballistic motion, maximum HSG occurs for preparation times that are offset with respect to the field crests, causing the distinct delay $\delta_{\mathrm{sc}}$ observed in Fig. $2 \mathrm{~d}$. The precise temporal structure seen in the experiment can be reproduced only if 

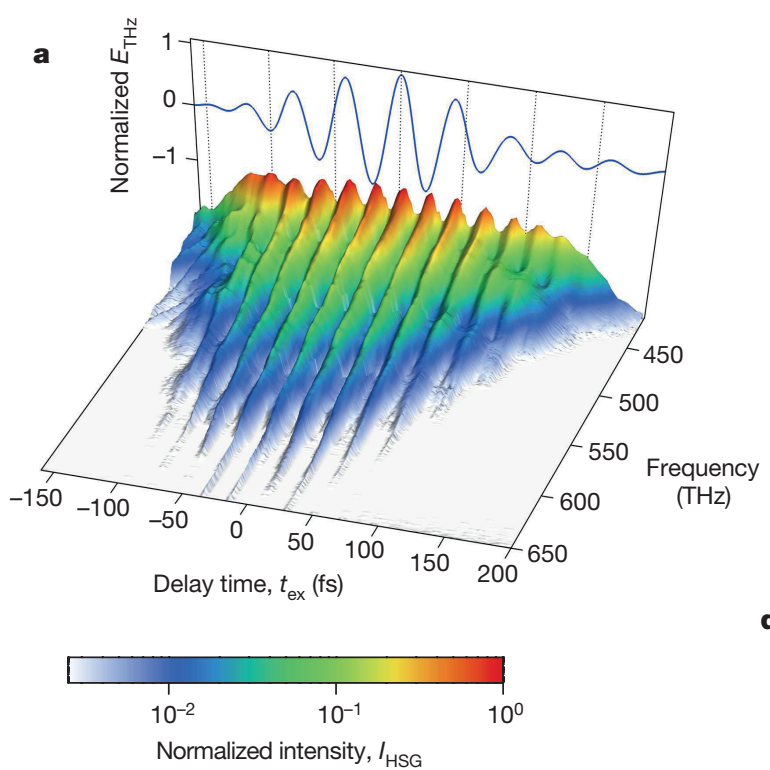

Figure 2 | Subcycle electron-hole recollisions. a, Spectrally resolved high-order sideband intensity $I_{\mathrm{HSG}}$ (colour scale) as a function of delay time $t_{\mathrm{ex}}$ between the $\mathrm{THz}$ driving field $\left(E_{\mathrm{THz}}\right.$, blue curve, see also $\left.\mathbf{b}\right)$ and a 10-fs interband excitation pulse. b, c, Measured (b) and calculated (c) high-order sideband intensity $I_{\mathrm{HSG}}$ (red, spectrally integrated between $435 \mathrm{THz}$ and $650 \mathrm{THz}$ ) on the same timescale as the driving waveform $\left(E_{\mathrm{THz}}\right.$, blue), which peaks with a global delay $\delta_{\text {global }}$ after $I_{\mathrm{HSG}}$. On a subcycle

excitation-induced dephasing ${ }^{27}$ is taken into account (see Methods section 'Effect of excitation-induced dephasing on HSG' and Extended Data Figs 4-6). By contrast, for 'bad' timing $t_{\mathrm{ex}}$, the electron and the hole are monotonically driven apart (Fig. 3c), suppressing collisions (see Fig. 3a). The situation is analogous to a cyclotron, in which electrons are effectively accelerated only if they are injected at the right phase of the alternating accelerator field.

Whereas the average intraband dynamics $\langle r\rangle$ resembles semiclassical trajectories (see Fig. 3c, d), the connection between quasiparticle collision and HSG requires a full quantum theory. Similarly to electronpositron pairs in vacuum, colliding electron-hole pairs in a solid are subject to pair annihilation, during which a high-energy photon may be emitted, leading to sideband signals; this microscopic annihilation dynamics manifests itself in $g_{\mathrm{coh}}(r)$ (Fig. 3h). After substantial spreading of $g_{\mathrm{coh}}(r)$, electron-hole collision is prompted by an abrupt termination of the correlation function due to pair annihilation. Subsequently, interference patterns occur among the residual fragments that survive the collision (see black ellipse in Fig. 3h). These distinct collision-induced annihilation features are absent in the case of 'bad' injection times, for which the coherence gradually abates owing to dephasing (Fig. 3g). The details of $g_{\mathrm{coh}}(r)$ are a direct manifestation of the many-body interactions governing the internal structure of the exciton, and are evident in the temporal and spectral response in HSG.

Detection of HSG, combined with precise preparation and acceleration, delivers an in-depth view of quasiparticles. As in particle colliders, one can expose the scattering products by systematic variation of key parameters, such as the strength, frequency and duration of the preparation and $\mathrm{THz}$ pulses, and their relative delay $t_{\mathrm{ex}}$. In Methods section 'Data landscape of quasiparticle colliders', we provide first examples of the ways in which to retrieve material-specific information such as the exciton binding energy, scattering times and Coulomb enhancement of the collisional cross-section via dedicated analyses of the multidimensional data landscape of $I_{\mathrm{HSG}}$. In particular, the collision events
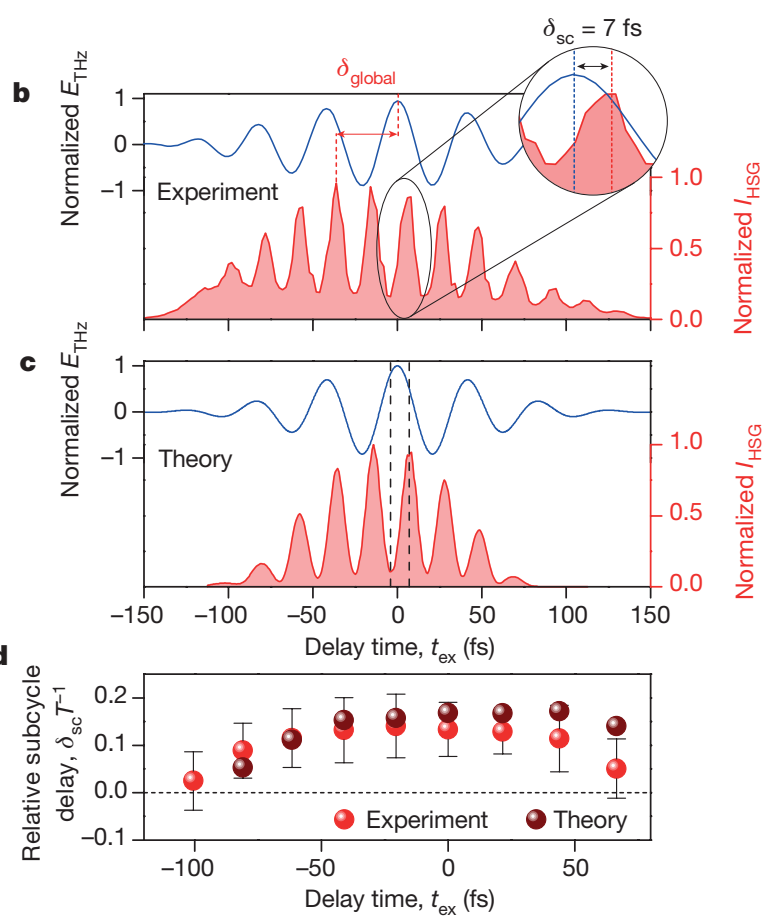

scale, the recorded sideband intensity (red) peaks at a distinct time delay $\delta_{\mathrm{sc}}$ after the nearest extrema of the driving waveform (see close-up in $\mathbf{b}$ ). d, Subcycle delay of $I_{\mathrm{HSG}}$ in units of the driving period $T$ for subsequent driving half-cycles at their respective delay times as measured (bright red spheres; error bars, standard deviation of $\delta_{\text {sc }}$ for 25 consecutive measurements) and calculated (dark red spheres). The horizontal black dashed line marks $\delta_{\mathrm{sc}} T^{-1}=0$.

recorded in our experiments bear the hallmarks of an exciton binding energy around $60 \mathrm{meV}$.

Well-defined initial momenta and relative spatial coordinates of the electrons and holes are prerequisites for controlled collisions. By contrast, the direction of acceleration in the $\mathrm{WSe}_{2}$ crystal is less relevant, as seen in Fig. 4: the high-order sideband spectra show virtually no dependence on the azimuthal in-plane angle $\varphi$ of the THz polarization. This behaviour is compatible with our theoretical expectation that coherent electron-hole wave packets in HSG remain rather close to $k=0$ (Fig. 3f). Both Coulomb attraction between electrons and holes and fast dephasing keep the coherent electron-hole excursions within a region in which the effective mass is approximately isotropic (see Fig. 1a). Additionally, HSG probes the interband polarization of colliding electrons and holes only at spatial overlap ${ }^{27}$ (inset of Fig. 4a).

This unique characteristic of the quasiparticle collider becomes particularly clear when we compare the dynamics with HHG in the same sample, which is achieved by applying the strong $\mathrm{THz}$ field without optical preparation of excitons. A typical high-harmonic spectrum exhibiting odd orders up to $n^{\prime}=47$ is depicted in Extended Data Fig. 7 . The intensity of HHG is smaller by approximately one order of magnitude than HSG for frequencies below $650 \mathrm{THz}$. Owing to the nonperturbative, non-resonant excitation of Bloch electrons with simultaneous acceleration by the strong-field bias ${ }^{20,21,24,26}$, the initial carrier distribution is smeared out throughout the entire Brillouin zone. Furthermore, coherent interband polarization can emit photons at any point in momentum space (see inset of Fig. 4b). This situation manifests itself by a critical dependence of the intensity and the shape of high-order harmonics on $\varphi$ (Fig. 4b), reflecting the six-fold symmetry of $\mathrm{WSe}_{2}$ in the layer plane.

In conclusion, we demonstrate the realization of quasiparticle collisions in the time domain. We excite, accelerate and collide coherent electron-hole pairs in $\mathrm{WSe}_{2}$ resulting in ultrabroad sideband emission. On a subcycle scale, we directly trace the retarded, ballistic wave-packet 

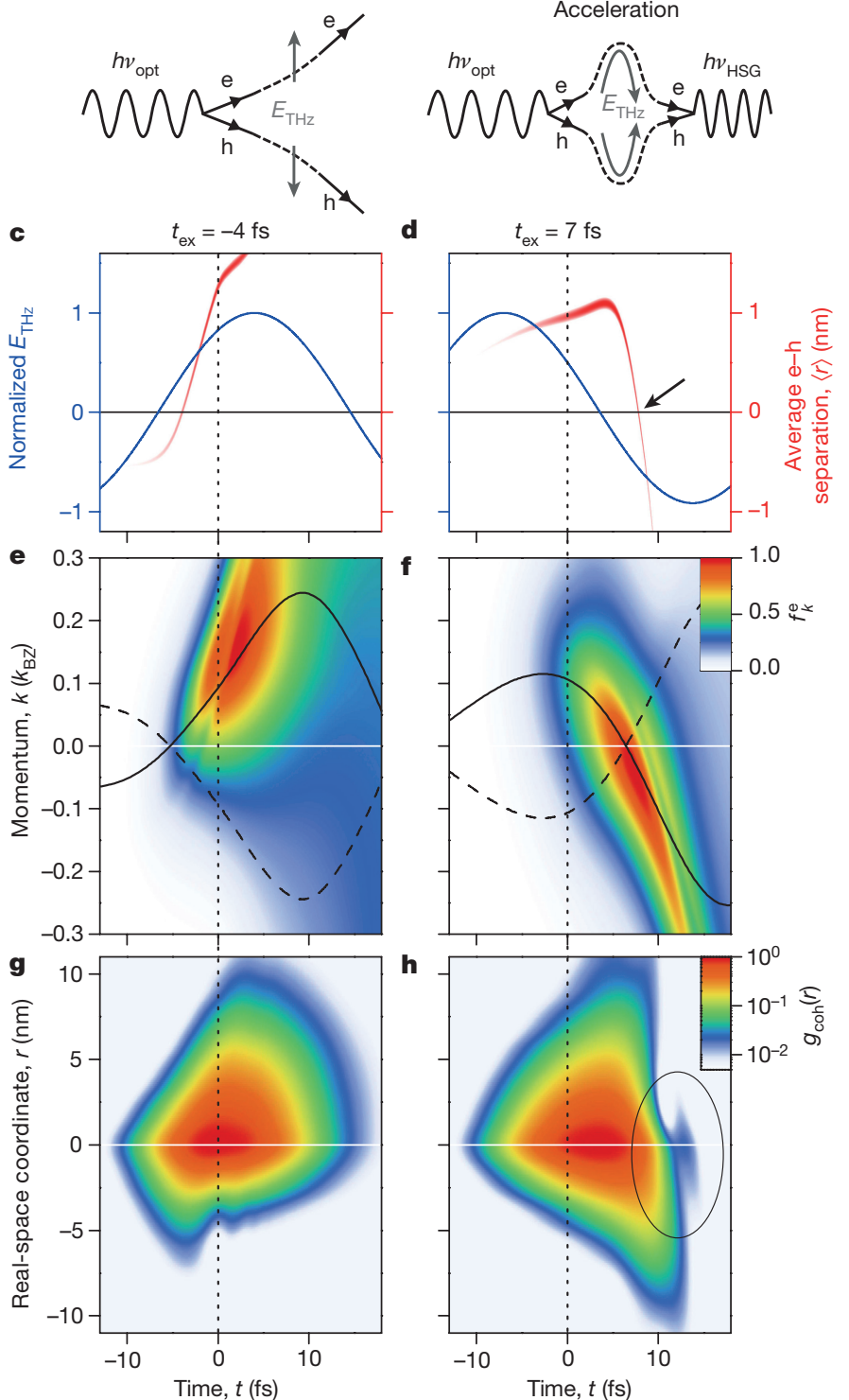

$\mathbf{h}$

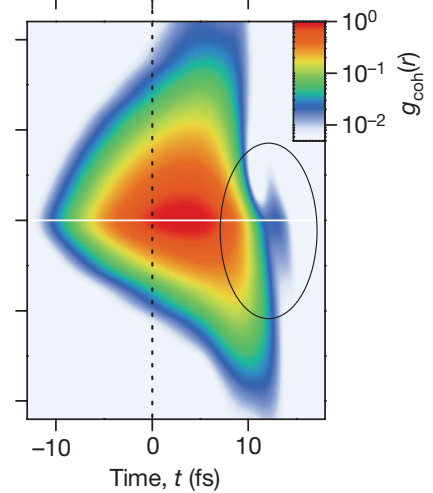

Figure 3 Quantum simulation of subcycle electron-hole collisions underlying HSG. a, b, Schematic Feynman diagrams depicting electronhole $(\mathrm{e}-\mathrm{h})$ pair creation by a near-infrared photon $\left(h \nu_{\mathrm{opt}}\right)$ and acceleration by $E_{\mathrm{THz}}$ : only for 'good' excitation times (b), the acceleration leads to a collision and annihilation of the electron-hole pair, thereby emitting a sideband photon $h \nu_{\text {HSG }}$. $\mathbf{c}, \mathbf{d}$, Trajectories (red, the intensity of the line represents the density of coherent excitons) tracing the real-time evolution (time $t$ ) of the mean electron-hole separation (weighted average of $g_{\mathrm{coh}}(r)$ ) for characteristic delays $t_{\mathrm{ex}}$ corresponding to minimum (c) and maximum (d) HSG emission (compare with Fig. 2c). Vertical black dotted lines highlight $t=0$, which marks the peak of the excitation pulse. Although electrons and holes are initially separated, they rapidly recollide (zero excursion marked by the black horizontal lines; time of recollision is highlighted by the black arrow in d) upon reversal of the driving field (blue curves), inducing a strong HSG signal (d). For 'bad' excitation times, the electron-hole separation increases monotonically, prohibiting recollisions (c). e, f, Occupation $f_{k}^{\mathrm{e}}$ (colour scale) of the first conduction band as a function of time $t$ and crystal momentum $k$ (in units of the wave vector $k_{\mathrm{BZ}}$ limiting the first Brillouin zone) for delays of $t_{\mathrm{ex}}=-4 \mathrm{fs}$ and $7 \mathrm{fs}$, respectively. Horizontal white lines mark $k=0$. Black solid (dashed) curves trace the weighted average excursion of electrons (holes) in reciprocal space. $\mathbf{g}, \mathbf{h}$, Coherent electron-hole correlation function $g_{\mathrm{coh}}(r)$ (colour scale) as a function of time $t$ and real-space coordinate $r$. Interference patterns occur after the abrupt collapse of coherence caused by electron-hole recollision (highlighted by the black ellipse in $\mathbf{h}$ ), and are absent for $t_{\mathrm{ex}}=-4 \mathrm{fs}(\mathrm{g})$. Horizontal white lines mark $r=0$. a

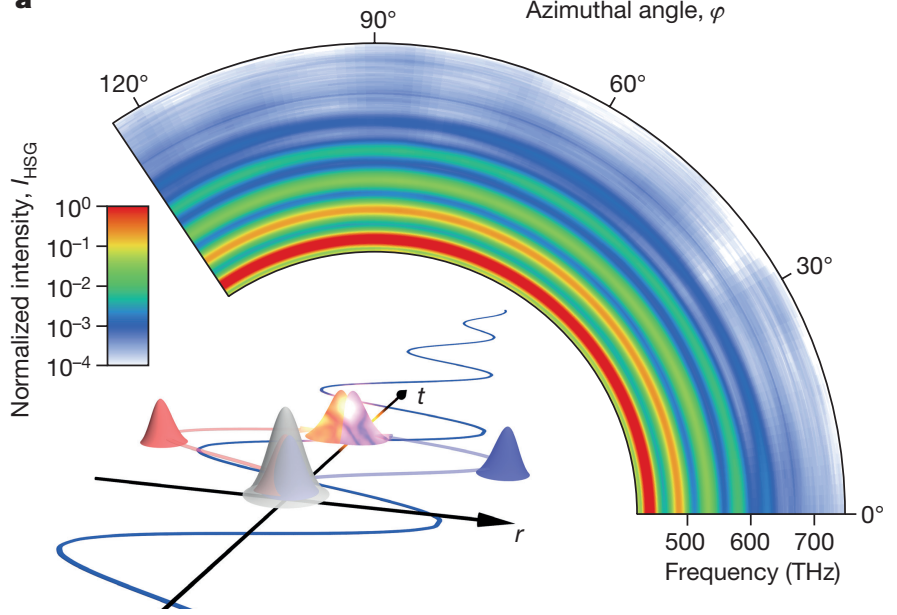

b

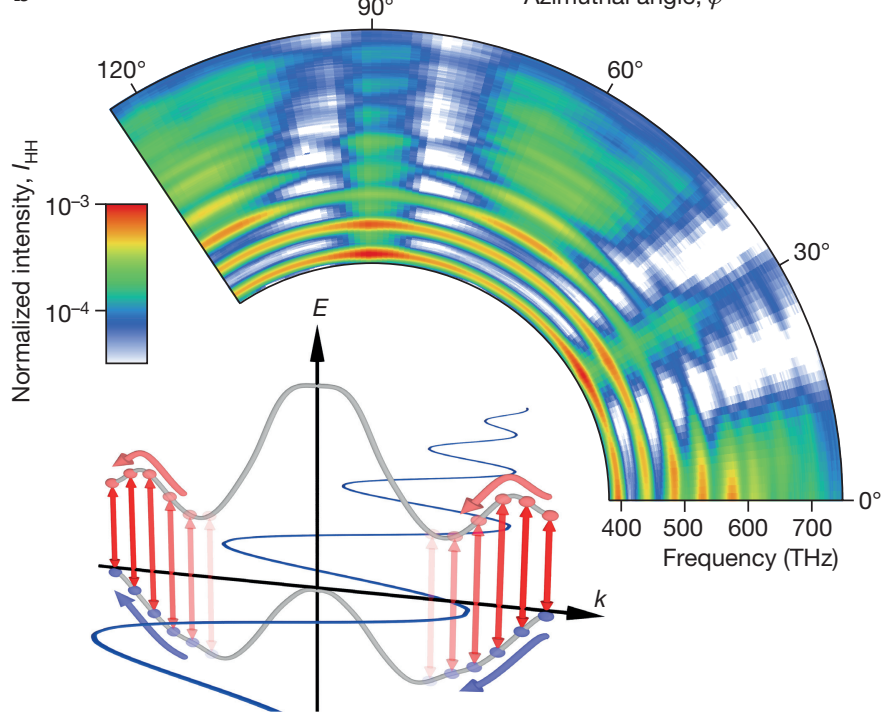

Figure 4 | Experimental comparison of high-order sideband and highorder harmonic generation. a, b, Spectrally resolved high-order sideband intensity $I_{\mathrm{HSG}}$ (a; narrow-band exciton preparation at a frequency of $392 \mathrm{THz}$ ) and high-order harmonic intensity $I_{\mathrm{HH}}(\mathbf{b})$ as a function of the azimuthal orientation (angle $\varphi$ ) of the $\mathrm{WSe}_{2}$ sample (normal incidence). Both intensity (colour) scales are normalized to the maximum HSG signal. Whereas high-order sidebands show virtually no dependence on $\varphi$, the high-harmonic spectra reflect the six-fold symmetry of WSe $\mathrm{W}_{2}$. a, Inset, real-space visualization of THz-driven electron-hole recollisions: upon excitation of a bound electron-hole pair (grey), the carriers (red and blue wave packets) are accelerated and recollided by the strong light field (blue) to recombine at $r=0$, giving rise to high-order sideband emission. $\mathbf{b}$, Inset, schematic of high-order harmonic generation: a polarization between valence and conduction band (grey) is induced by a strong multi- $\mathrm{THz}$ field (blue) and simultaneously accelerated within the bands (red and blue spheres, curved red and blue arrow). During this process, the coherent interband polarization is continuously modified (red vertical arrows).

dynamics and retrieve key information such as the exciton binding energy, scattering times and the Coulomb enhancement of the collisional cross-section. As in conventional particle accelerators, our optical excitation pulse prepares electron and hole wave packets with uncertainty-limited momenta and relative spatial coordinates. Yet their absolute position is defined only to within the relatively large focal spot size, which may be overcome in future near-field experiments by preparing excitons in a nanometre-sized interaction region. Both in the quasiparticle collider and in its large-scale counterpart, intrinsic characteristics of the collision become accessible by detecting the concomitantly emitted high-frequency radiation. The high 
density of quasiparticles in solids helps to generate relatively intense, all-coherent, ultrabroadband radiation, which may pave the way to new sub-femtosecond light sources; furthermore, the subcycle modulation of HSG may facilitate information processing at optical-clock rates. Large-scale colliders have enabled the detailed study of elementary particles. Likewise, we anticipate that $\mathrm{THz}$-driven, real-time collisions-operating on much smaller, widely tunable, length and energy scales-will be key to studying complex quasiparticles in numerous applications of modern materials science; examples include excitons, trions, biexcitons or dropletons in quasitwo-dimensional atomic monolayer systems, Dirac-like quasiparticles in graphene and topological insulators, or polarons in materials featuring strong electron-phonon coupling. This scheme may also be extended to optically excited quasiparticles in unconventional superconductors, and could help to resolve some of the outstanding questions in condensed matter research.

Online Content Methods, along with any additional Extended Data display items and Source Data, are available in the online version of the paper; references unique to these sections appear only in the online paper.

\section{Received 13 November 2015; accepted 18 March 2016.}

1. Rutherford, E. The scattering of $\alpha$ and $\beta$ particles by matter and the structure of the atom. Philos. Mag. Ser. 6 21, 669-688 (1911).

2. Wyatt, T. High-energy colliders and the rise of the standard model. Nature $\mathbf{4 4 8 ,}$ 274-280 (2007)

3. Chemla, D. S. \& Shah, J. Many-body and correlation effects in semiconductors. Nature 411, 549-557 (2001).

4. Almand-Hunter, A. E. et al. Quantum droplets of electrons and holes. Nature 506, 471-475 (2014).

5. Basov, D. N., Averitt, R. D., van der Marel, D., Dressel, M. \& Haule, K. Electrodynamics of correlated electron materials. Rev. Mod. Phys. 83, 471-541 (2011).

6. Rossi, F. \& Kuhn, T. Theory of ultrafast phenomena in photoexcited semiconductors. Rev. Mod. Phys. 74, 895-950 (2002)

7. Corkum, P. B. Plasma perspective on strong-field multiphoton ionization. Phys. Rev. Lett. 71, 1994-1997 (1993).

8. Lewenstein, M., Balcou, P., Ivanov, M. Y., L'Huillier, A. \& Corkum, P. B. Theory of high-harmonic generation by low-frequency laser fields. Phys. Rev. A 49, 2117-2132 (1994).

9. Paul, P. M. et al. Observation of a train of attosecond pulses from high harmonic generation. Science 292, 1689-1692 (2001).

10. Corkum, P. B. \& Krausz, F. Attosecond science. Nature Phys. 3, 381-387 (2007).

11. Li, W. et al. Time-resolved dynamics in $\mathrm{N}_{2} \mathrm{O}_{4}$ probed using high harmonic generation. Science 322, 1207-1211 (2008).

12. Smirnova, O. et al. High harmonic interferometry of multi-electron dynamics in molecules. Nature 460, 972-977 (2009).

13. Neppl, S. et al. Direct observation of electron propagation and dielectric screening on the atomic length scale. Nature 517, 342-346 (2015).

14. Breuer, J. \& Hommelhoff, P. Laser-based acceleration of nonrelativistic electrons at a dielectric structure. Phys. Rev. Lett. 111, 134803 (2013).
15. Wimmer, L. et al. Terahertz control of nanotip photoemission. Nature Phys. 10, 432-436 (2014).

16. Nanni, E. A. et al. Terahertz-driven linear electron acceleration. Nature Commun. 6, 8486 (2015).

17. Kono, J. et al. Resonant terahertz optical sideband generation from confined magnetoexcitons. Phys. Rev. Lett. 79, 1758-1761 (1997).

18. Zaks, B., Liu, R. B. \& Sherwin, M. S. Experimental observation of electron-hole recollisions. Nature 483, 580-583 (2012)

19. Liu, R. B. \& Zhu, B. F. High-order THz-sideband generation in semiconductors. AIP Conf. Proc. 893, 1455-1456 (2007).

20. Ghimire, S. et al. Observation of high-order harmonic generation in a bulk crystal. Nature Phys. 7, 138-141 (2011)

21. Schubert, O. et al. Sub-cycle control of terahertz high-harmonic generation by dynamical Bloch oscillations. Nature Photon. 8, 119-123 (2014).

22. Luu, T. T. et al. Extreme ultraviolet high-harmonic spectroscopy of solids. Nature 521, 498-502 (2015)

23. Vampa, G. et al. Linking high-harmonics from gases and solids. Nature $\mathbf{5 2 2}$, 462-464 (2015)

24. Hohenleutner, M. et al. Real-time observation of interfering crystal electrons in high-harmonic generation. Nature 523, 572-575 (2015).

25. Shah, J. Ultrafast Spectroscopy of Semiconductors and Semiconductor Nanostructures (Springer, Berlin 1999).

26. Higuchi, T., Stockman, M. I. \& Hommelhoff, P. Strong-field perspective on high-harmonic radiation from bulk solids. Phys. Rev. Lett. 113, 213901 (2014).

27. Kira, M. \& Koch, S. W. Semiconductor Quantum Optics (Cambridge University Press, Cambridge, 2012).

28. Poellmann, C. et al. Resonant internal quantum transitions and femtosecond radiative decay of excitons in monolayer $\mathrm{WSe}_{2}$. Nature Mater. 14, 889-893 (2015).

29. Xu, X., Wang, Y., Xiao, D. \& Heinz, T. F. Spin and pseudospins in layered transition metal dichalcogenides. Nature Phys. 10, 343-350 (2014).

30. Arora, A. et al. Excitonic resonances in thin films of $\mathrm{WSe}_{2}$ : from monolayer to bulk material. Nanoscale 7, 10421-10429 (2015).

Acknowledgements We thank $\mathrm{H}$. Banks for critical reading of the manuscript The work in Regensburg was supported by the European Research Council through grant number 305003 (QUANTUMsubCYCLE) and the Deutsche Forschungsgemeinschaft (through grant number HU 1598/2-1 and GRK 1570), and the work in Marburg by the Deutsche Forschungsgemeinschaft (through SFB 1083, SPP 1840 and grant numbers KI 917/2-2, KI 917/3-1). The work in Santa Barbara was supported by the National Science Foundation (through grant number DMR 1405964).

Author Contributions F.L., M.H., M.S.S., U.H., M.K. and R.H. conceived the study. F.L., M.H., C.P.S. and R.H. carried out the experiment and analysed the data. C.P., P.N., T.K. and C.S. provided, processed and characterized the samples. U.H., J.T.S., S.W.K. and M.K. developed the quantum-mechanical model and carried out the computations. All authors discussed the results and contributed to the writing of the manuscript.

Author Information Reprints and permissions information is available at www.nature.com/reprints. The authors declare no competing financial interests. Readers are welcome to comment on the online version of the paper. Correspondence and requests for materials should be addressed to R.H. (rupert.huber@physik.uni-regensburg.de) or M.K. (mackillo.kira@physik. uni-marburg.de). 


\section{METHODS}

Experimental set-up. Intense, phase-locked waveforms in the far- to midinfrared spectral region (multi-terahertz range) are generated by difference frequency mixing of two spectrally detuned, near-infrared pulse trains from a dual optical parametric amplifier pumped by a titanium-sapphire laser amplifier ${ }^{21,24}$. The few-cycle transients feature peak fields of up to approximately $1 \mathrm{~V} \AA^{-1}$ (ref. 31). A super-continuum source based on filamentation in an yttrium aluminium garnet crystal delivers ultrabroadband white-light pulses covering the whole nearinfrared and visible range. Both pulse trains are collinearly superimposed with an indium-tin-oxide-coated beam splitter and focused onto the tungsten diselenide sample under normal incidence using a gold-coated parabolic mirror. A mechanical delay stage in the excitation beam path allows us to temporally delay the pulses with respect to each other.

The sample under study is a 60 -nm-thick sheet of $\mathrm{WSe}_{2}$ that has been cleaved using a viscoelastic gel film and placed on a diamond substrate grown by chemical vapour deposition. For resonant, narrow-band excitation of the excitonic polarization in $\mathrm{WSe}_{2}$, optical band-pass filters featuring a transmittance bandwidth of $10 \mathrm{~nm}$ are used (see Fig. 1d). For the time-resolved measurements, the bandwidth of the excitation pulse is adjusted in the Fourier plane of a prism compressor, which enables the required pulse duration of $10 \mathrm{fs}$. Spectral components of the excitation pulse above $400 \mathrm{THz}$ are filtered out to avoid the resonant generation of a high density of unbound electron-hole pairs. All experiments were performed at room temperature and in ambient air. No sample degradation was observed during the whole course of experiments.

The spot sizes of the excitation pulse and the THz pulse at the sample are $22 \mu \mathrm{m}$ and $85 \mu \mathrm{m}$, respectively (intensity full-width at half-maximum). The generated high-order sideband intensity $I_{\mathrm{HSG}}$ and the high-order harmonic intensity $I_{\mathrm{HH}}$ are recorded with a spectrograph featuring a cooled silicon CCD camera. All spectra are corrected for the grating efficiency and the quantum efficiency of the detector.

The pulse energy of the narrowband excitation amounts to approximately $0.1 \mathrm{~nJ}$ in our current experiments. A comparison of the spectral components of the excitation and the sideband signals yields an energy conversion efficiency of approximately $0.1 \%$ in the 60 -nm-thick WSe ${ }_{2}$ sample for the applied excitation fluence (approximately $25 \mu \mathrm{J} \mathrm{cm}^{-2}$ ). Therefore, we estimate the energy in the high-order sideband pulse train as roughly $0.1 \mathrm{pJ}$.

Replacing the $\mathrm{WSe}_{2}$ sample by a $6.5-\mu \mathrm{m}$-thick zinc telluride (ZnTe) detector crystal allows for electro-optic detection of the driving field. To this end, we carefully place the crystal into the $\mathrm{THz}$ focus at the same position and account for the complex detector response ${ }^{32}$. The delay time $t_{\mathrm{ex}}$ between the $\mathrm{THz}$ waveform and the near-infrared excitation pulse enables a direct temporal correlation of the driving waveform with the recorded trace of high-order sideband emission. To describe the microscopic dynamics following exciton creation, a second timescale $t$ is introduced, which describes the real time evolving for a fixed value of $t_{\mathrm{ex}}$ as shown, for instance, in Fig. 3.

Microscopic model of HSG. A complete quantum description of optical ${ }^{33,34}$ and $\mathrm{THz}$ excitations ${ }^{21,24,35,36}$ of solids follows from the semiconductor Bloch equations ${ }^{27}$ (SBEs). Compared to earlier HSG models ${ }^{19}$, the SBEs fully include manybody effects beyond isolated electron-hole pairs, the interplay of HSG versus HHG, non-parabolic dispersions, and dephasing and relaxation processes. The SBEs are

$$
\begin{aligned}
i \hbar \frac{\partial}{\partial t} p_{\boldsymbol{k}} & =\tilde{\varepsilon}_{\boldsymbol{k}} p_{\boldsymbol{k}}-\hbar \Omega_{\boldsymbol{k}}(t)\left(1-f_{\boldsymbol{k}}^{\mathrm{e}}-f_{-\boldsymbol{k}}^{\mathrm{h}}\right)+i|e| \boldsymbol{E}_{\mathrm{THz}}(t) \cdot \nabla_{\boldsymbol{k}} p_{\boldsymbol{k}}+\Gamma_{\boldsymbol{k}} \\
\hbar \frac{\partial}{\partial t} f_{\boldsymbol{k}}^{\mathrm{e}} & =-2 \operatorname{Im}\left[\hbar \Omega_{\boldsymbol{k}}(t) p_{\boldsymbol{k}}^{*}\right]+|e| \boldsymbol{E}_{\mathrm{THz}}(t) \cdot \nabla_{\boldsymbol{k}} f_{\boldsymbol{k}}^{\mathrm{e}}+\Gamma_{\boldsymbol{k}}^{\mathrm{e}} \\
\hbar \frac{\partial}{\partial t} f_{\boldsymbol{k}}^{\mathrm{h}} & =-2 \operatorname{Im}\left[\hbar \Omega_{-\boldsymbol{k}}(t) p_{-\boldsymbol{k}}^{*}\right]+|e| \boldsymbol{E}_{\mathrm{THz}}(t) \cdot \nabla_{-\boldsymbol{k}} f_{-\boldsymbol{k}}^{\mathrm{h}}+\Gamma_{\boldsymbol{k}}^{\mathrm{h}}
\end{aligned}
$$

in which $p_{k}^{*}$ is the complex conjugate of the microscopic interband polarization $p_{k}$ and $f_{k}^{\mathrm{e}}\left(f_{k}^{\mathrm{h}}\right)$ defines the electron (hole) occupation in a conduction-band (valenceband) state with a crystal momentum $\hbar \boldsymbol{k}$ ( $\hbar$ is the reduced Planck constant). The equations also contain the strength of the field coupling via $\Omega_{k}$ and the renormalized single-particle energies $\tilde{\varepsilon}_{\boldsymbol{k}}$; the elementary charge is denoted by $e$, and scattering contributions are considered by $\Gamma_{k}, \Gamma_{k}^{\mathrm{e}}$, and $\Gamma_{k}^{\mathrm{h}}$. Physically, $p_{k}$ defines the transition amplitude of exciting an electron from one band to another while $\hbar \boldsymbol{k}$ is conserved. As an optical field $\boldsymbol{E}_{\text {opt }}(t)$ induces such a transition, it eventually gen erates occupations $f_{k}^{\mathrm{e}}$ and $f_{k}^{\mathrm{h}}$. The presented SBEs can be generalized in a straightforward manner for multiple bands ${ }^{21,37,38}$.

As an inverse process, electrons recombine with holes via photon emission. The coherent part of this process yields radiative decay of the polarization ${ }^{27}$ and the corresponding emission spectrum is ${ }^{35} I=|\omega \boldsymbol{P}(\omega)+i \boldsymbol{J}(\omega)|^{2}$ at energy $\hbar \omega$. The Fourier transforms of the macroscopic polarization $\boldsymbol{P}(t)=\sum_{\boldsymbol{k}} \boldsymbol{d}_{\mathrm{cv}}(\boldsymbol{k}) p_{\boldsymbol{k}}+$ c.c. (in which c.c. represents the complex conjugate) and the macroscopic current
$\boldsymbol{J}(t)=\sum_{\lambda, \boldsymbol{k}} \boldsymbol{j}_{\boldsymbol{k}}^{\lambda} f_{\boldsymbol{k}}^{\lambda}$ contain the dipole and current matrix elements $\boldsymbol{d}_{\mathrm{cv}}(\boldsymbol{k})$ and $\boldsymbol{j}_{\boldsymbol{k}}^{\lambda}=\frac{|e|}{\hbar} \boldsymbol{\nabla}_{\boldsymbol{k}} \varepsilon_{\boldsymbol{k}}^{\lambda}$, respectively. The currents in a given band $\lambda=\mathrm{e}, \mathrm{h}$ are defined by its energy dispersion $\varepsilon_{\boldsymbol{k}}^{\lambda}$.

Because electrons and holes are charged particles, they experience a strong Coulomb force, which creates non-trivial many-body effects among $p_{k}, f_{k}^{\mathrm{e}}$ and $f_{k}^{\mathrm{h}}$ during their finite lifetimes. For example, the strength of the field excitation becomes renormalized

$$
\hbar \Omega_{\boldsymbol{k}}(t)=\boldsymbol{d}_{\mathrm{cv}}(\boldsymbol{k}) \cdot \boldsymbol{E}(t)+\sum_{\boldsymbol{k}^{\prime}} V_{\boldsymbol{k}-\boldsymbol{k}^{\prime}} \boldsymbol{P}_{\boldsymbol{k}^{\prime}}
$$

by the Coulomb matrix element $V_{k}$, while its un-normalized part is a product of $\boldsymbol{d}_{\mathrm{cv}}(\boldsymbol{k})$ and the total field $\boldsymbol{E}=\boldsymbol{E}_{\mathrm{opt}}+\boldsymbol{E}_{\mathrm{THz}}$ containing also the THz field $\boldsymbol{E}_{\mathrm{THz}}$. The Coulomb sum within $\Omega_{\boldsymbol{k}}(t)$ results in excitonic resonances in the polarization ${ }^{27}$. The single-particle transition energy $\varepsilon_{k}$ becomes renormalized too

$$
\widetilde{\varepsilon}_{\boldsymbol{k}}=\varepsilon_{\boldsymbol{k}}-\sum_{\boldsymbol{k}^{\prime}} V_{\boldsymbol{k}-\boldsymbol{k}^{\prime}}\left(f_{\boldsymbol{k}^{\prime}}^{\mathrm{e}}+f_{\boldsymbol{k}^{\prime}}^{\mathrm{h}}\right)
$$

Additionally, phonon, photon and Coulomb interactions yield scattering contributions $\Gamma_{k}, \Gamma_{k}^{\mathrm{e}}$ and $\Gamma_{k}^{\mathrm{h}}$ due to high-order correlations ${ }^{33}$. We include microscopic scattering with the approximation $\Gamma_{k}=-i \frac{\hbar}{T_{2}(\boldsymbol{k})} p_{\boldsymbol{k}}$, which includes excitationinduced dephasing (EID) via the $\boldsymbol{k}$-dependent scattering time $T_{2}(\boldsymbol{k})$, as explained in Methods section 'Effect of excitation-induced dephasing on HSG' Microscopically, $\Gamma_{k}^{\mathrm{e}}$ and $\Gamma_{k}^{\mathrm{h}}$ induce a relaxation of electron and hole distributions, which we implement using a phenomenological relaxation towards a symmetric carrier distribution via $\Gamma_{k}^{\lambda}=-\frac{1}{2 \tau}\left(f_{k}^{\lambda}+f_{-\boldsymbol{k}}^{\lambda}\right)$ on a timescale defined by $\tau$ (ref. 24) In our computations, $\tau$ is chosen to be $6 \mathrm{fs}$.

Our experiments show that high-order sideband generation (HSG) excites carriers only in the vicinity of the $\mathrm{K}$ point where $\mathrm{WSe}_{2}$ electrons and holes can be described with an effective mass approximation. To determine the general aspects of the interplay between HSG and high-harmonic generation (HHG), we use a onedimensional (1D) two-band model ${ }^{35}$, and adjust $V_{\boldsymbol{k}}$ to produce a binding energy $E_{\mathrm{B}}=60 \mathrm{meV}$ (corresponding to $14.5 \mathrm{THz}$ ) for the $1 s$-exciton state ${ }^{30}$. We match electrons and holes to produce the same effective mass $m_{\mathrm{e}}=m_{\mathrm{h}}=0.52 m_{0}$ (ref. 39), with the free electron mass $m_{0}$, close to the K point. We solve the closed system of coupled differential equations (1) numerically using a Runge-Kutta algorithm.

We have confirmed that a two-dimensional (2D) computation produces essentially the same HSG results. Note that bulk WSe $e_{2}$ features a layered structure where electron-hole motion is free only within each $2 \mathrm{D}$ layer. However, only a $1 \mathrm{D}$ computation is numerically feasible to fully include HHG, Bloch oscillations and a non-parabolicity in the dispersion $\varepsilon_{\boldsymbol{k}}$. The $1 \mathrm{D}$ computations also confirm that HHG is an order of magnitude weaker than HSG, as in the experiment.

Coherent electron-hole pair-correlation function. The excitations underlying HSG can be investigated in real space by following the electron-hole paircorrelation function

$$
g_{\mathrm{eh}}(\boldsymbol{r}) \equiv\left\langle\hat{\rho}_{\mathrm{e}}(\boldsymbol{r}) \hat{\rho}_{\mathrm{h}}(\mathbf{0})\right\rangle
$$

containing the operators for electron and hole densities $\hat{\rho}_{\mathrm{e}}$ and $\hat{\rho}_{\mathrm{h}}$, respectively. As discussed in ref. 33, the envelope of $\hat{\rho}_{\mathrm{e}}(\boldsymbol{r})$ and $\hat{\rho}_{\mathrm{h}}(\mathbf{0})$ determines the ways in which electrons move with respect to holes on length scales larger than the unit cell. The resulting $g_{\text {eh }}(r)$ defines the conditional probability of finding an electron at position $r$ when a hole is at the origin.

To identify the coherent electron-hole acceleration, it is convenient to separate incoherent and coherent contributions from $g_{\mathrm{eh}}(\boldsymbol{r})$. Following the derivation in ref. 33, we find $g_{\text {eh }}(\boldsymbol{r})=g_{\text {inc }}(\boldsymbol{r})+g_{\text {coh }}(\boldsymbol{r})$, with coherent part $g_{\text {coh }}(\boldsymbol{r})=|p(\boldsymbol{r})|^{2}$ and incoherent part $g_{\text {inc }}(\boldsymbol{r})=\rho_{\mathrm{e}} \rho_{\mathrm{h}}+\Delta g_{\mathrm{eh}}(\boldsymbol{r})$, which is proportional to the product of electron and hole densities $\left(\rho_{\mathrm{e}}\right.$ and $\left.\rho_{\mathrm{h}}\right)$, to the lowest order. The formation of pairwise, incoherent correlations generates an additional contribution $\Delta g_{\mathrm{eh}}(\boldsymbol{r})$. However, $g_{\mathrm{coh}}(\boldsymbol{r})$ is independent of correlation formation and is exclusively defined by the coherent polarization $p(\boldsymbol{r})$, which is given by the Fourier transform of $p_{k}$ and describes the relative motion of electron-hole coherences. In other words, $g_{\text {coh }}(\boldsymbol{r})$ defines the way in which coherently generated electrons are distributed with respect to the holes in real space. Because the SBEs fully include the manybody aspects of the polarization dynamics $p_{k}, g_{\text {coh }}(r)$ generalizes the descriptions of following a single electron-hole pair during $\mathrm{HSG}^{19,40}$.

Effect of excitation-induced dephasing on HSG. To quantify collision dynamics under different conditions, Extended Data Fig. $6 \mathrm{~b}$ compares the mean wave vector

$$
\langle\boldsymbol{k}\rangle=\frac{\sum_{k} \boldsymbol{k}\left|p_{\boldsymbol{k}}\right|^{2}}{\sum_{\boldsymbol{k}}\left|p_{\boldsymbol{k}}\right|^{2}}
$$


of the electron-hole wave packet for a 10-fs-long (red curve) and a 100-fs-long (black curve) optical excitation pulse centred at $t_{\mathrm{ex}}=7 \mathrm{fs}$, while the dephasing is kept constant $\left(T_{2}=12 \mathrm{fs}\right.$ ). The THz waveform (Extended Data Fig. 6a) is identical for both optical excitations. The respective envelope of the excitation pulse is shown as the grey (yellow) shaded area for the $100-\mathrm{fs}(10-\mathrm{fs})$ pulse.

We observe that the electron-hole wave packets are accelerated to much higher momentum states in the case of the long (100-fs) excitation pulse compared to the short (10-fs) excitation pulse. As discussed in the main text, a zero crossing of $\langle\boldsymbol{k}\rangle$ enables collisions and annihilation of optically generated coherent excitons. For the short excitation pulse, the magnitude of $\left|p_{k}\right|^{2}$ substantially drops after the recollision. Therefore, the motion of residual coherent electron-hole pairs is indicated by the red dashed curve in Extended Data Fig. 6b, c, whereas the dominant $\left|p_{k}\right|^{2}$ motion is plotted as red solid curve. For the long excitation pulse, the excitonic polarization $\left|p_{k}\right|^{2}$ is generated continuously, such that it is not diminished substantially by the collisions. The corresponding mean wave vector $\langle\boldsymbol{k}\rangle$ is plotted as a black curve in Extended Data Fig. 6b, c.

Applying a constant dephasing time $T_{2}$ implies that all excitonic components of the polarization decay with an identical rate. However, the Coulomb interaction scatters polarization with the excited electron-hole distributions, yielding a strong exciton-state-dependent $\mathrm{EID}^{33}$. More specifically, the 1 s-exciton state (which is tightly bound around the $\mathrm{K}$ point) features lower scattering rates than other exciton states that spread out in $\boldsymbol{k}$, which is included by introducing a $\boldsymbol{k}$-dependent dephasing time $T_{2}(\boldsymbol{k})$ as shown in the inset of Extended Data Fig. 4a: for low $\boldsymbol{k}$, $T_{2}(\boldsymbol{k})$ is $12 \mathrm{fs}$, whereas it decreases to $2 \mathrm{fs}$ at elevated $\boldsymbol{k}$. Comparably fast dephasing times have also been observed in previous studies ${ }^{24,41,42}$. Additionally, the large temporal modulations in the HSG intensity as shown in Fig. 2a imply dephasing times $T_{2}$ that are substantially shorter than one half-cycle of the $\mathrm{THz}$ driving field $\left(T_{2} \ll T / 2=22 \mathrm{fs}\right)$.

To determine the effect of EID on electron-hole collisions, Extended Data Fig. 6c shows $\langle\boldsymbol{k}\rangle$ for the momentum-dependent dephasing $T_{2}(\boldsymbol{k})$. By comparing the black curves (100-fs-long excitation) in Extended Data Fig. 6b and c, we conclude that EID reduces the maximum excursion by roughly a factor of 3 compared to the constant dephasing result. The mean momentum trajectory $\langle\boldsymbol{k}\rangle$ for the short (10-fs) excitation pulse is less affected by EID, owing to the reduced maximum displacement.

Extended Data Fig. 4 compares the measured, time-integrated HSG spectra (shaded area) with computations including EID (black curves), a constant dephasing $T_{2}=3.2 \mathrm{fs}$ (Extended Data Fig. 4a, red curve), and $T_{2}=4 \mathrm{fs}$ (Extended Data Fig. 4b, blue curve), respectively. We observe that only calculations taking EID into account explain the height of all observed sidebands. More specifically, the simulations using a constant dephasing achieve good agreement only for either low-order sidebands ( $n<8, T_{2}=4 \mathrm{fs}$ ) or sidebands above $600 \mathrm{THz}\left(T_{2}=3.2 \mathrm{fs}\right)$. In numbers, $T_{2}=4 \mathrm{fs}\left(T_{2}=3.2 \mathrm{fs}\right.$ ) overestimates (underestimates), for example, the tenth-order (fourth-order) sideband by a factor of $1.9(1.7)$. This result implies that EID induces non-trivial spectral imprints on the HSG spectrum. These modulations result from the anharmonic electron-hole acceleration identified in Extended Data Fig. 6c.

In general, the SBE approach systematically describes the creation of coherent excitons, quasiparticle acceleration, collision-related annihilation, the interplay between HSG and HHG contributions, and the influence of many-body effects such as EID or phonon scattering processes as suggested by ref. 43 . Only some aspects of these effects can be quantitatively analysed using either a semiclassical approach or a quantum description of a single electron-hole pair. Because manybody effects can induce drastic changes to HSG and HHG, it is clear that the SBE analysis is needed to properly parameterize any simplified model.

Influence of excitation fluence. With increasing optical fluence $\Phi$, the measured sideband intensity increases linearly (Extended Data Fig. 2a); however, the modulation depth of time-resolved traces of $I_{\mathrm{HSG}}$ is found to decrease (Extended Data Fig. 2b). This feature may be most intuitively explained by considering that a growing density of excitons may instantly lead to phase-space filling and broadening causing less-well-defined initial conditions for electron-hole recollisions. By contrast, a potential increase in dephasing rates does not seem to be a dominant factor, because faster dephasing should result in an increase in the modulation depth-the opposite effect of what we observe. Additionally, the subcycle delay $\delta_{\mathrm{sc}}$, which is sensitively dependent on the dephasing time $T_{2}$, does not change substantially with varying excitation fluence, implying that major density-driven effects on $T_{2}$ have not yet built up (Extended Data Fig. 2c). However, such excitation-induced effects are important for the case of narrowband excitation analysed in Fig. 1 and Extended Data Fig. 4.

Data landscape of quasiparticle colliders. In regular optical spectroscopy, system resonances of interest are often imbedded within the spectral range of the applied pump field. Our quasiparticle collider spectrally separates the relevant features from the pump as distinct sidebands. Additionally, each sideband order can contain different aspects of the collisions. As in conventional colliders, the scattering-generated radiation produces a wealth of information about the (quasi) particles. Here, we study the HSG intensity $I_{\mathrm{HSG}}$ as a function of the HSG frequency $\nu_{\mathrm{HSG}}$, the field strength $E_{\lambda}$, the duration $\tau_{\lambda}$ and the central frequency $\nu_{\lambda}$ of the optical preparation pulse $(\lambda=\mathrm{opt})$ and the THz waveform $(\lambda=\mathrm{THz})$, and their mutual delay $t_{\mathrm{ex}}$, generating at least eight-dimensional datasets: $I_{\mathrm{HSG}}\left(\nu_{\mathrm{HSG}}, E_{\mathrm{THz}}\right.$, $\left.\tau_{\mathrm{THz}}, \nu_{\mathrm{THz}}, E_{\mathrm{opt}}, \tau_{\mathrm{opt}}, \nu_{\mathrm{opt}}, t_{\mathrm{ex}}\right)$. As is typical for colliders, such a wealth of data reveals details of colliding quasiparticles only through a dedicated identification process. For example, Fig. 3 demonstrates how changing $t_{\mathrm{ex}}$ yields a direct detection of the electron-hole recollision. We will next illustrate a few additional possibilities to extract exciton-related details from the HSG scattering data.

As shown in Extended Data Fig. 4, broadening of sideband resonances is directly related to exciton decay rates, which depend on the excitation parameters. This effect is often referred to as $\operatorname{EID}^{44,45}$, which can be controlled, for example, by changing $E_{\text {opt }}$ (ref. 34). We have also confirmed that the exciton binding energy $E_{\mathrm{B}}$ strongly influences the overall strength of the HSG signal. For example, a tenfold increase of $E_{\mathrm{B}}$ amplifies $I_{\mathrm{HSG}}$ by roughly the same amount as a result of Coulomb enhancement of the collisional cross-section. Hence, a variety of material- and excitation-dependent exciton features may be deduced by quantitatively analysing the strength and width of $I_{\mathrm{HSG}}$ resonances.

By changing the optical pump frequency $\nu_{\text {opt }}$ one can deduce differential changes in HSG spectra that sensitively monitor material details. We propose measuring a $2 \mathrm{D} I_{\mathrm{HSG}}\left(\nu_{\mathrm{HSG}}, \nu_{\mathrm{opt}}\right)$ set to follow spectral changes at the second-order sideband and to define a normalized spectrum

$$
I\left(\nu, \nu_{\mathrm{opt}}\right) \equiv \frac{I_{\mathrm{HSG}}\left(\nu_{\mathrm{HSG}}-\nu_{\mathrm{opt}}, \nu_{\mathrm{opt}}\right)}{I_{2 \mathrm{SB}}}
$$

in which $\nu=\nu_{\mathrm{HSG}}-\nu_{\mathrm{opt}}$ is the frequency scale centred around $\nu_{\mathrm{opt}}$ and $I_{2 \mathrm{SB}}=I_{\mathrm{HSG}}\left(\nu_{\mathrm{opt}}+2 \nu_{\mathrm{THz}}, \nu_{\mathrm{opt}}\right)$ is the peak intensity of the second-order sideband. This normalization produces spectra that do not depend on the absolute intensity scale - a quantity that is difficult to determine experimentally for a wide range of frequencies $\nu_{\text {opt }}$. From the difference of two spectra measured with pump frequencies $\nu_{\text {opt }} \mp \Delta \nu_{\text {opt }} / 2$, we deduce a finite differential

$$
\Delta I\left(\nu, \nu_{\mathrm{opt}}\right)=\frac{1}{\Delta \nu_{\mathrm{opt}}}\left[I\left(\nu, \nu_{\mathrm{opt}}-\Delta \nu_{\mathrm{opt}} / 2\right)-I\left(\nu, \nu_{\mathrm{opt}}+\Delta \nu_{\mathrm{opt}} / 2\right)\right]
$$

which approaches the derivative $\frac{\partial I}{\partial \nu_{\mathrm{opt}}}$ in the limit $\Delta \nu_{\mathrm{opt}} \rightarrow 0$.

Extended Data Fig. 8a, b shows computed $\Delta I\left(\nu, \nu_{\text {opt }}\right)$ for two $1 s$-exciton binding energies, $E_{\mathrm{B}}=60 \mathrm{meV}$ and $E_{\mathrm{B}}=600 \mathrm{meV}$, respectively, when $h \nu_{\mathrm{opt}}$ is expressed in terms of the detuning $\Delta_{\text {opt }}$ with respect to the $1 s$-exciton energy $h \nu_{1 s}$ as $h \nu_{\text {opt }}=h \nu_{1 s}+\Delta_{\text {opt }}$. These contours produce spectral information that differs greatly for the two exciton binding energies, demonstrating that $\Delta I\left(\nu, \nu_{\text {opt }}\right)$ exposes material-dependent properties. For $E_{\mathrm{B}}=60 \mathrm{meV}, \Delta I\left(\nu, \nu_{\text {opt }}\right)$ exhibits dominantly negative changes for $\Delta_{\mathrm{opt}}<0$, implying that $I_{\mathrm{HSG}}$ decreases for negative detuning. Because $\Delta_{\text {opt }}>0$ yields mainly positive $\Delta I\left(\nu, \nu_{\text {opt }}\right)$, a clear dispersive shape is observed around the resonant excitation $\left(\Delta_{\mathrm{opt}}=0\right)$. For $E_{\mathrm{B}}=600 \mathrm{meV}$, a positive island appears within the $\Delta_{\mathrm{opt}}<0$ region, which implies that $I_{\mathrm{HSG}}$ can be enhanced also for negative $\Delta_{\mathrm{opt}}$ in contrast to the $E_{\mathrm{B}}=60 \mathrm{meV}$ case. Additionally, $\Delta_{\mathrm{opt}}>0$ produces a new feature because the positive islands split into a double-peaked structure for $E_{\mathrm{B}}=600 \mathrm{meV}$.

To analyse the quantitative dependence of $\Delta I\left(\nu, \nu_{\mathrm{opt}}\right)$ on $E_{\mathrm{B}}$, Extended Data Fig. $8 \mathrm{c}, \mathrm{d}$ shows slices of $\Delta I\left(\nu, \nu_{\text {opt }}\right)$ at fixed values of $h\left(\nu-\nu_{2 \mathrm{SB}}\right)= \pm 16 \mathrm{meV}$, respectively, for three different binding energies $E_{\mathrm{B}}=60 \mathrm{meV}$ (black curve), $240 \mathrm{meV}$ (red curve) and $600 \mathrm{meV}$ (blue curve). The slice at $-16 \mathrm{meV}$ shows only one positive peak for $\Delta_{\text {opt }}>0$ and remains negative at $\Delta_{\text {opt }}<0$ for $E_{\mathrm{B}}=60 \mathrm{meV}$ (black curve). Increasing the exciton binding energy gradually creates a new maximum within the region of negative detuning. This transition from a single- to a double-peaked structure can be exploited to assign the exciton binding energy as shown below. Monitoring the slices at $h\left(\nu-\nu_{2 \mathrm{SB}}\right)=+16 \mathrm{meV}$ reveals a smooth, narrow, single-peaked structure close to $\Delta_{\text {opt }}=0$ for $E_{\mathrm{B}}=60 \mathrm{meV}$ (black curve). By contrast, higher binding energies produce a more structured shape.

To construct differential spectra $\Delta I\left(\nu, \nu_{\text {opt }}\right)$ experimentally, we measure HSG spectra for narrowband pump pulses at different frequencies $\nu_{\text {opt }}$ (see Fig. 1b, d) while varying $t_{\mathrm{ex}}$. Extended Data Fig. $9 \mathrm{c}$ shows the resulting intensity map $I_{\mathrm{HSG}}\left(\nu, \nu_{\mathrm{opt}}, t_{\mathrm{ex}}\right)$ for resonant excitation $h \nu_{\mathrm{opt}}=h \nu_{1 s}$; the corresponding computation is shown in Extended Data Fig. 9e. Both experiment and theory show the same spectral structure, including $t_{\mathrm{ex}}$-dependent changes in the intensity and shape. A similar level of agreement is realized also for non-resonant excitations (not shown), paving the way to thorough theory-experiment comparison of the differential spectra. 
On the basis of Extended Data Fig. 8, we expect the strongest dependence of $\Delta I\left(\nu, \nu_{\text {opt }}\right)$ on $E_{\mathrm{B}}$ for a finite detuning $\Delta_{\text {opt }}$ from the $1 s$-exciton resonance. We therefore compare experimental traces $\Delta I\left(\nu, \nu_{\mathrm{opt}}\right)$ at $t_{\mathrm{ex}}=-20 \mathrm{fs}$ for three different detunings $\Delta_{\text {opt }}=-75 \mathrm{meV}$ (shaded area), $-29 \mathrm{meV}$ (black curve) and $27 \mathrm{meV}$ (red curve) in Extended Data Fig. 9a. A negative $\Delta_{\text {opt }}$ produces a dispersive line shape to $\Delta I\left(\nu, \nu_{\text {opt }}\right)$, that is, a dip followed by a zero crossing and a peak, which implies a shift of the underlying HSG intensity with a change in $\nu_{\mathrm{opt}}$. For positive $\Delta_{\text {opt }}$ a double-peaked structure indicates a broadening of the HSG peaks. For a binding energy of $E_{\mathrm{B}}=60 \mathrm{meV}$, these line shapes are well reproduced by the theoretical computations using the same detuning $\Delta_{\text {opt }}$ and excitation conditions as in the experiment, as shown in Extended Data Fig. $9 \mathrm{~b}$.

Extended Data Fig. 9d, f shows the corresponding calculated differentials $\Delta I\left(\nu, \nu_{\text {opt }}\right)$ when the exciton binding energy is increased to $E_{\mathrm{B}}=240 \mathrm{meV}$ and $E_{\mathrm{B}}=600 \mathrm{meV}$, respectively. Whereas $\Delta_{\mathrm{opt}}=-29 \mathrm{meV}$ (black curve) and $\Delta_{\text {opt }}=27 \mathrm{meV}$ (red curve) produce a similar shape as for $E_{\mathrm{B}}=60 \mathrm{meV}$, the peak amplitude of the differential curve for $\Delta_{\text {opt }}=-75 \mathrm{meV}$ (shaded area) greatly decreases for an intermediate binding energy of $E_{\mathrm{B}}=240 \mathrm{meV}$ and turns into a negative dip for the largest binding energy. This structural change in $\Delta I\left(\nu, \nu_{\mathrm{opt}}\right)$ can be used to deduce the exciton binding energy. We find that the experimental data are consistent with $E_{\mathrm{B}}=60 \pm 20 \mathrm{meV}$, which agrees well with values reported in literature ${ }^{30,46,47}$

31. Sell, A., Leitenstorfer, A. \& Huber, R. Phase-locked generation and field-resolved detection of widely tunable terahertz pulses with amplitudes exceeding $100 \mathrm{MV} / \mathrm{cm}$. Opt. Lett. 33, 2767-2769 (2008).

32. Gallot, G. \& Grischkowsky, D. Electro-optic detection of terahertz radiation. J. Opt. Soc. Am. B 16, 1204-1212 (1999)

33. Kira, M. \& Koch, S. W. Many-body correlations and excitonic effects in semiconductor spectroscopy. Prog. Quantum Electron. 30, 155-296 (2006).
34. Smith, R. P. et al. Extraction of many-body configurations from nonlinear absorption in semiconductor quantum wells. Phys. Rev. Lett. 104, 247401 (2010).

35. Golde, D., Kira, M., Meier, T. \& Koch, S. W. Microscopic theory of the extremely nonlinear terahertz response of semiconductors. Phys. Status Solidi B 248, 863-866 (2011)

36. Danielson, J. R. et al. Interaction of strong single-cycle terahertz pulses with semiconductor quantum wells. Phys. Rev. Lett. 99, 237401 (2007).

37. Girndt, A., Jahnke, F. Knorr, A., Koch, S. W. \& Chow, W. W. Multi-band Bloch equations and gain spectra of highly excited II-VI semiconductor quantum wells. Phys. Status Solidi B 202, 725-739 (1997).

38. Berger, C. et al. Novel type-Il material systems for laser applications in the near-infrared regime. AlP Advances 5, 047105 (2015).

39. Ramasubramaniam, A. Large excitonic effects in monolayers of molybdenum and tungsten dichalcogenides. Phys. Rev. B 86, 115409 (2012).

40. Yan, J.-Y. Theory of excitonic high-order sideband generation in semiconductors under a strong terahertz field. Phys. Rev. B 78, 075204 (2008).

41. Vu, Q. T. et al. Light-induced gaps in semiconductor band-to-band transitions. Phys. Rev. Lett. 92, 217403 (2004).

42. Vampa, G. et al. Theoretical analysis of high-harmonic generation in solids. Phys. Rev. Lett. 113, 073901 (2014).

43. Banks, $\mathrm{H}$. et al. Terahertz electron-hole recollisions in GaAs/AlGaAs quantum wells: robustness to scattering by optical phonons and thermal fluctuations. Phys. Rev. Lett. 111, 267402 (2013)

44. Wang, H. et al. Transient nonlinear optical response from excitation induced dephasing in GaAs. Phys. Rev. Lett. 71, 1261-1264 (1993).

45. Peyghambarian, N. et al. Blue shift of the exciton resonance due to excitonexciton interactions in a multiple-quantum-well structure. Phys. Rev. Lett. 53, 2433-2436 (1984)

46. Beal, A. R. \& Liang, W. L. Excitons in $2 \mathrm{H}-\mathrm{WSe}_{2}$ and $3 R-W S_{2}$. J. Phys. C 9 , 2459-2466 (1976)

47. Mitioglu, A. A. et al. Optical investigation of monolayer and bulk tungsten diselenide $\left(\mathrm{WSe}_{2}\right)$ in high magnetic fields. Nano Lett. 15, 4387-4392 (2015). 


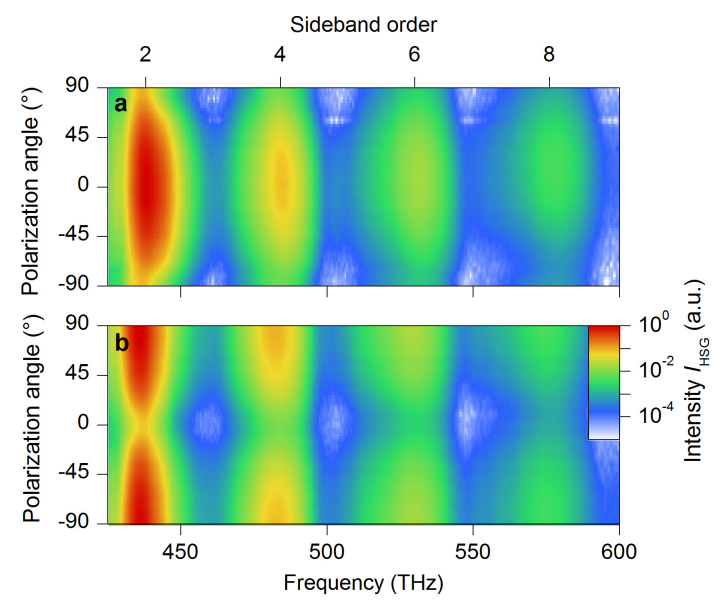

Extended Data Figure 1 | Polarization of high-order sidebands. a, b, False-colour plot of the spectral intensity of high-order sidebands (generated under resonant, spectrally narrow, optical excitation) of orders two to eight as a function of their polarization for horizontal (a) and

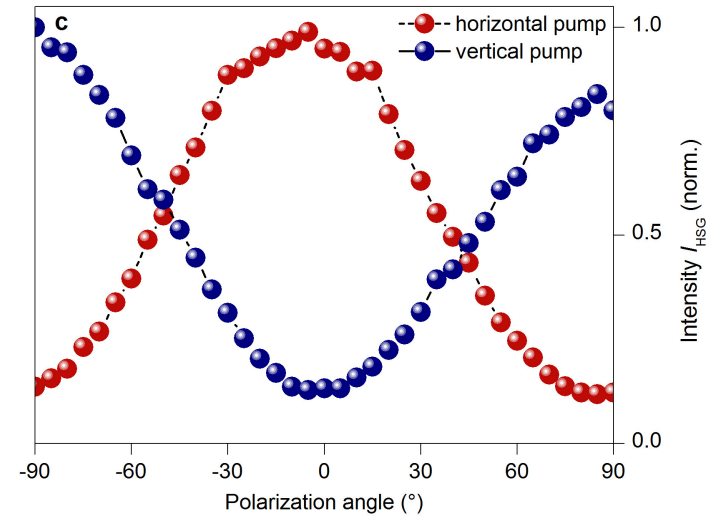

vertical (b) polarization of the interband excitation pulse. The polarization angle is defined such that $0^{\circ}$ corresponds to a horizontally polarized excitation. c, Spectrally integrated sideband intensity as a function of the polarization angle. 


\section{RESEARCH LETTER}
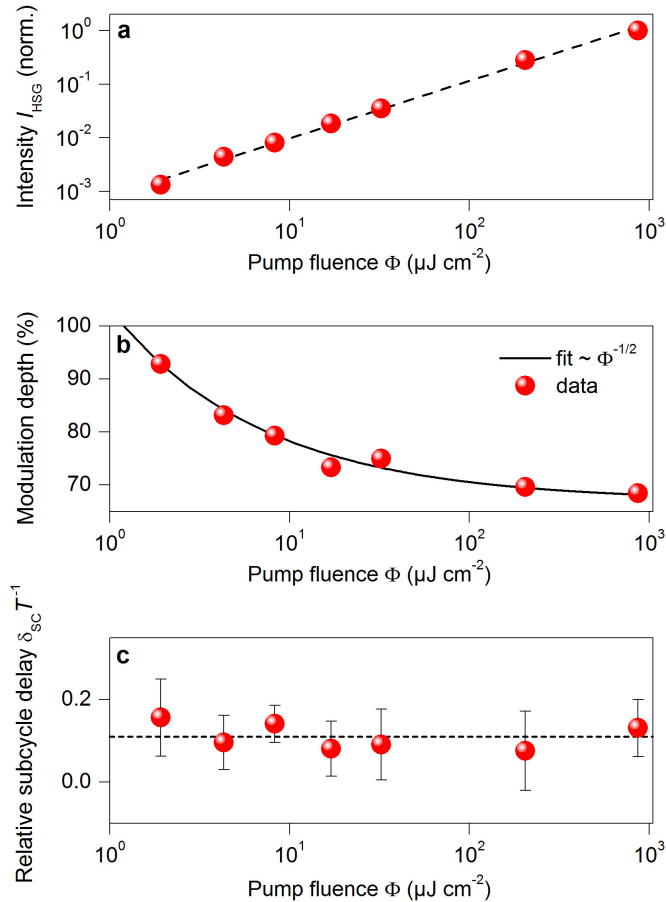

Extended Data Figure $2 \mid$ Pump-fluence dependence of time-resolved high-order sideband generation. a, The measured high-order sideband intensity $I_{\mathrm{HSG}}$ (spectrally and temporally integrated, red spheres) scales linearly with the pump fluence $\Phi$, as indicated by a guide to the eye (black dashed line). b, The modulation depth of the spectrally integrated temporal trace of $I_{\mathrm{HSG}}$ (red spheres, see Fig. 2b) decreases with increasing pump fluence, closely following a fit (black curve) proportional to the inverse square-root of the pump fluence. c, By contrast, the relative subcycle delay $\delta_{\mathrm{sc}} T^{-1}$ does not substantially change with increasing pump fluence. Red spheres represent the average of $\delta_{\mathrm{sc}} T^{-1}$ over the nine dominant, consecutive, high-order sideband peaks (see Fig. 2d) for different pump fluences; error bars indicate their standard deviation. The black dashed line marks the mean value of the displayed data points. 


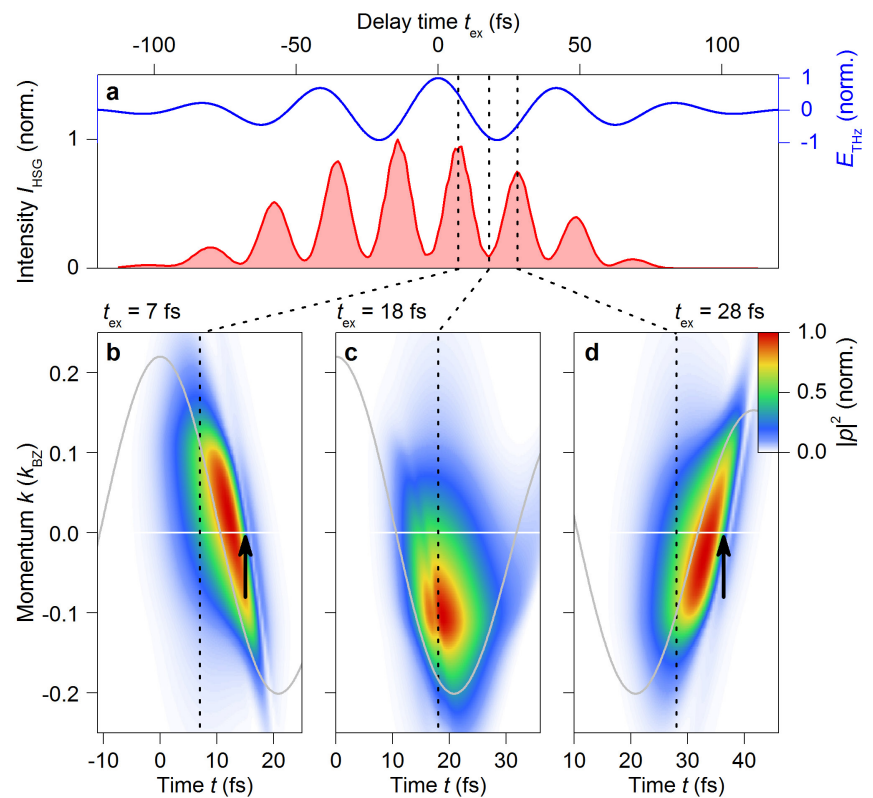

Extended Data Figure 3 | Coherent excitonic polarization dynamics in $\boldsymbol{k}$ space. a, Computed high-order sideband intensity $I_{\mathrm{HSG}}$ (red shaded area) and driving waveform (blue). Vertical black dotted lines highlight characteristic delay times $t_{\mathrm{ex}}$ at extrema of $I_{\mathrm{HSG}}$. $\mathbf{b}-\mathbf{d}$, Coherent interband polarization $|p|^{2}$ (colour scale) as a function of time $t$ and reciprocal space coordinate $k$ (in units of the wave vector $k_{\mathrm{BZ}}$ limiting the first Brillouin zone) for distinct delay times $t_{\mathrm{ex}}$ according to maximum (b, $t_{\mathrm{ex}}=7 \mathrm{fs}$; d, $t_{\mathrm{ex}}=28 \mathrm{fs}$ ) and minimum (c, $t_{\mathrm{ex}}=18 \mathrm{fs}$ ) HSG emission. Horizontal white lines mark $k=0$. The driving field is depicted as grey curves and the recollision times (see Fig. 3d) are highlighted by black arrows. 


\section{RESEARCH LETTER}
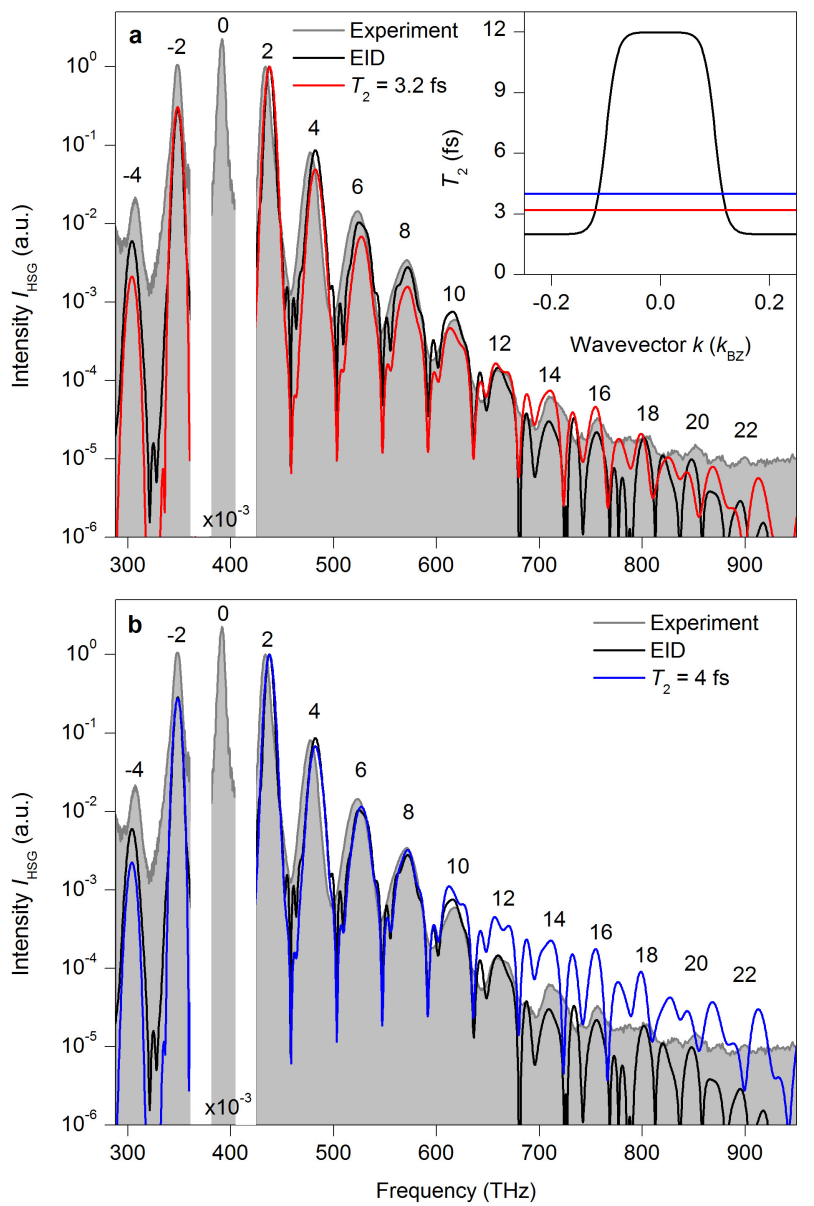

Extended Data Figure 4 Influence of dephasing on high-order sideband generation. a, b, The measured sideband spectrum (shaded area) is compared with computations using constant dephasing times of $T_{2}=3.2 \mathrm{fs}$ (a, red curve), $T_{2}=4 \mathrm{fs}$ (b, blue curve) and a momentumdependent dephasing model as presented in Fig. 1 (black curves; EID, excitation-induced dephasing). The sideband orders $n$ are indicated above the relevant peaks. All spectra are normalized to the sideband peak corresponding to $n=2$. The inset in a depicts the corresponding dephasing times $T_{2}(\boldsymbol{k})$ as a function of the wave vector $\boldsymbol{k}$; the red (blue) horizontal line indicates a constant decay level $T_{2}=3.2 \mathrm{fs}\left(T_{2}=4 \mathrm{fs}\right)$. 

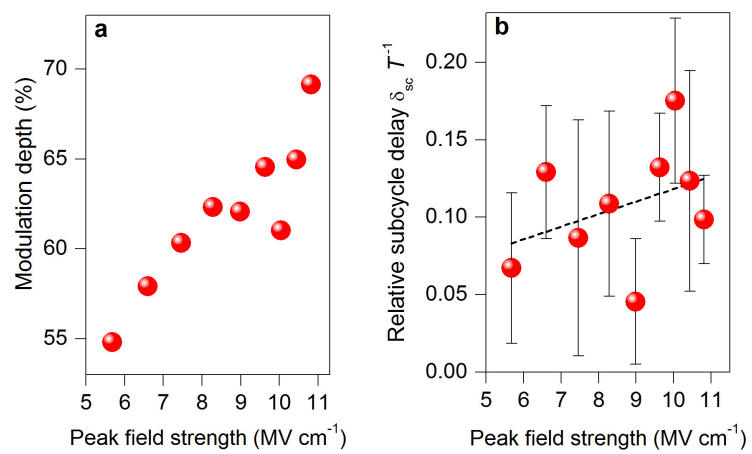

Extended Data Figure 5 | Field scaling of time-resolved high-order sideband generation. a, Scaling of the modulation depth (see discussion in Methods section 'Influence of excitation fluence') of temporal traces of high-order sideband intensity with the driving peak field strength. An increase in the modulation depth suggests a faster dephasing $T_{2}$. b, Subcycle delay $\delta_{\mathrm{sc}}$ in units of the driving period $T$ averaged over the eight most-dominant sideband peaks as a function of the peak field.

The error bars represent the standard deviation of the eight peaks and the dashed line depicts a linear fit to the data points. 


\section{RESEARCH LETTER}
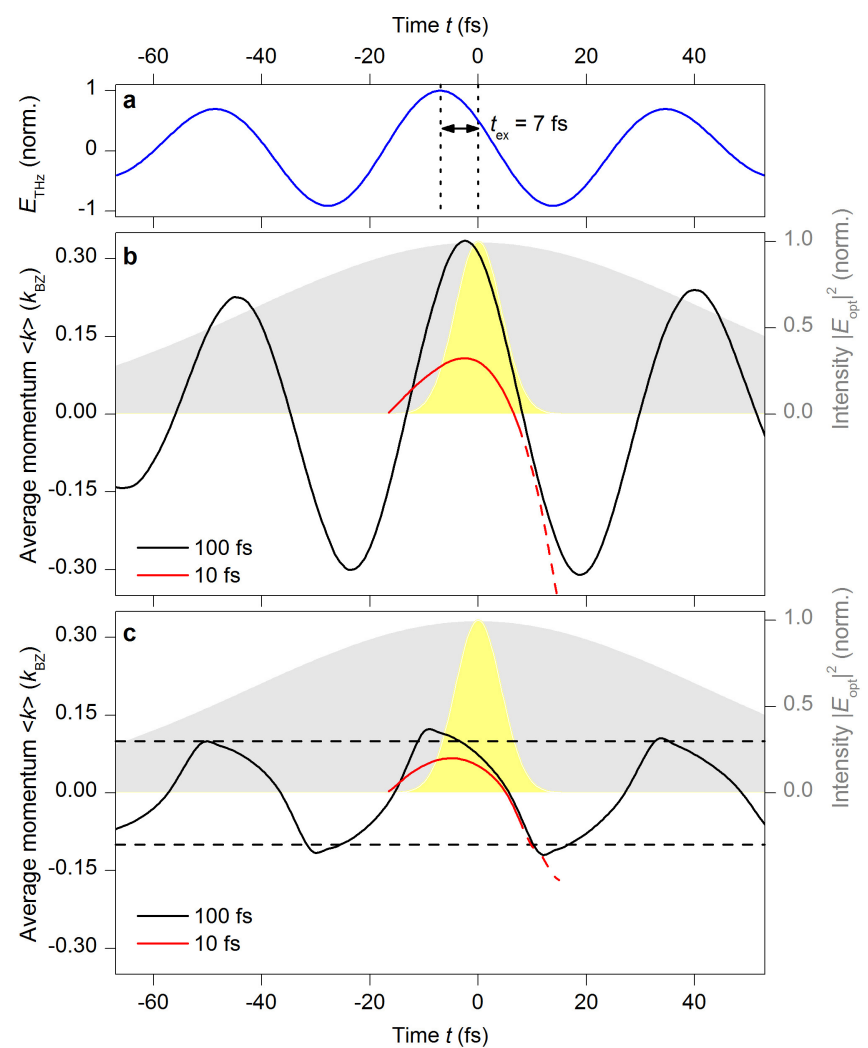

Extended Data Figure 6 | Influence of excitation-induced dephasing.

a, Terahertz waveform used in the computations. b, c, Mean electron excursion $\langle\boldsymbol{k}\rangle$ calculated with a constant dephasing $T_{2}=12 \mathrm{fs}(\mathbf{b})$ and excitation-induced dephasing (c) for a delay of $t_{\mathrm{ex}}=7 \mathrm{fs}$ (see vertical dotted lines in a). The shaded areas indicate the intensity envelopes of the excitation pulse with a full-width at half-maximum of $10 \mathrm{fs}$ (yellow) and $100 \mathrm{fs}$ (grey). The corresponding mean momenta $\langle\boldsymbol{k}\rangle$ are shown as red and black curves, respectively. The red dashed curves show $\langle\boldsymbol{k}\rangle$ after the electron-hole collision. The dashed horizontal lines in $\mathbf{c}$ mark the positions in $\boldsymbol{k}$ space where the scattering time $T_{2}(\boldsymbol{k})$ switches from slow to fast dephasing. 


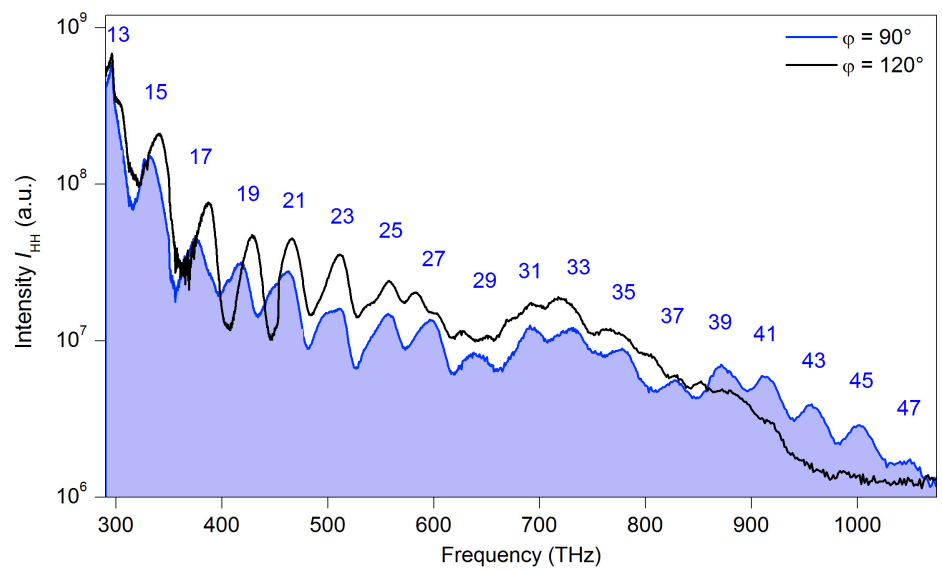

Extended Data Figure 7 | High-order harmonic generation in tungsten diselenide. An intensity spectrum (blue shaded area) of THz-driven highorder harmonic generation in 60-nm-thick WSe $\mathrm{W}_{2}$ shows distinct peaks at frequency $\nu_{\mathrm{THz}}=22.3 \mathrm{THz}$ (peak electric field in air, $21 \mathrm{MV} \mathrm{cm}^{-1}$; $\varphi=90^{\circ}$ ). An intensity spectrum for $\varphi=120^{\circ}$ is also shown as a black odd orders $n^{\prime}=13$ to $n^{\prime}=47$ (indicated by numerals) of the fundamental curve. The spectral intensity has been corrected for the grating efficiency and for the quantum efficiency of the spectrograph used. 


\section{RESEARCH LETTER}
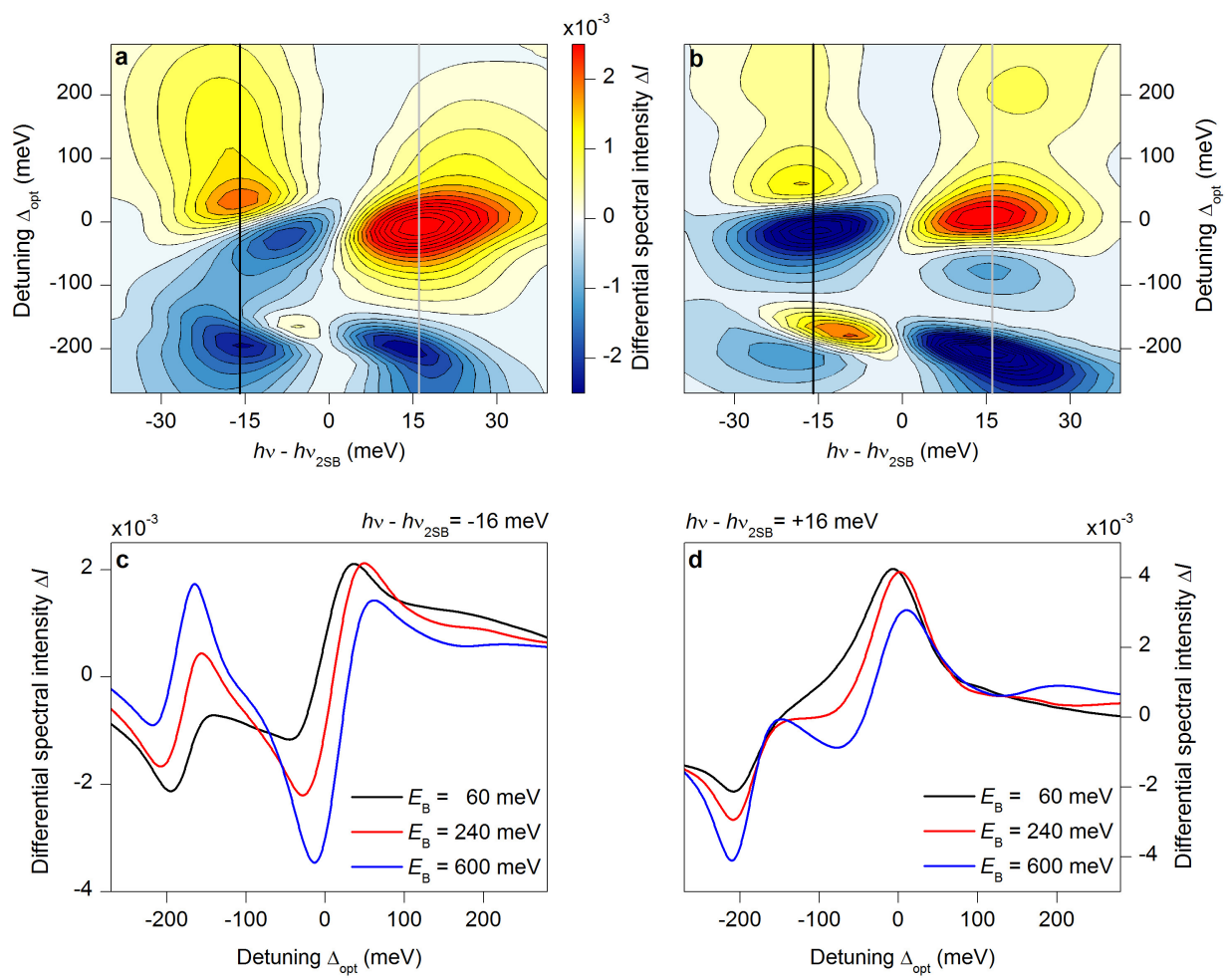

Extended Data Figure 8 Differential sideband spectroscopy.

a, $\mathbf{b}$, Contours of differential spectra $\Delta I\left(\nu, \nu_{\text {opt }}\right)$ (colour scale) as a function of $h\left(\nu-\nu_{2 \mathrm{SB}}\right)$ and detuning $\Delta_{\mathrm{opt}}$ computed for an excitonic binding energy of $E_{\mathrm{B}}=60 \mathrm{meV}(\mathbf{a})$ and $E_{\mathrm{B}}=600 \mathrm{meV}(\mathbf{b})$. The black and grey vertical lines mark the positions of the slices shown in $\mathbf{c}$ and $\mathbf{d}$,

respectively. c, d, Snapshots of $\Delta I\left(\nu, \nu_{\text {opt }}\right)$ at fixed values of $\left|h\left(\nu-\nu_{2 \mathrm{SB}}\right)\right|=$ $16 \mathrm{meV}$ below (c) and above (d) the second-order sideband peak for three different binding energies $E_{\mathrm{B}}=60 \mathrm{meV}$ (black curves), $E_{\mathrm{B}}=240 \mathrm{meV}$ (red curves) and $E_{\mathrm{B}}=600 \mathrm{meV}$ (blue curves). 

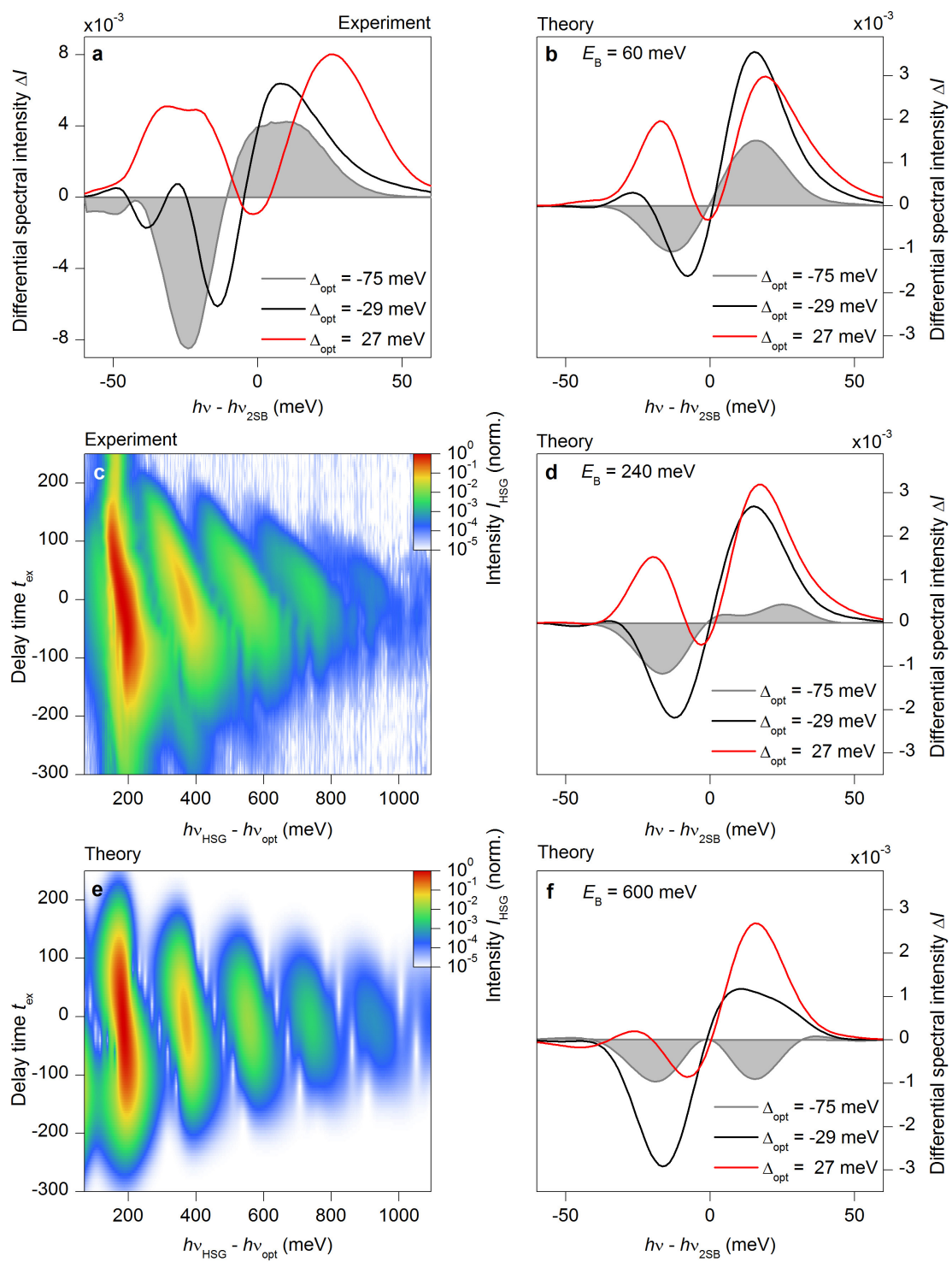

Extended Data Figure 9 | Quantitative analysis of the binding energy. a, Measured differential spectra $\Delta I\left(\nu, \nu_{\text {opt }}\right)$ for three different detunings $\Delta_{\text {opt }}=-75 \mathrm{meV}$ (shaded area), $\Delta_{\text {opt }}=-29 \mathrm{meV}$ (black curve) and

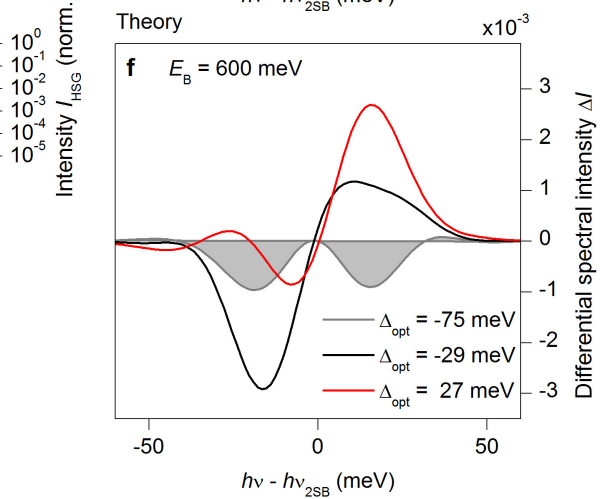

$\Delta_{\mathrm{opt}}=27 \mathrm{meV}$ (red curve) as functions of $\nu$, centred at the position of the second sideband $\nu_{2 \mathrm{SB}} \cdot \mathbf{b}, \mathbf{d}, \mathbf{f}$, Computed differential spectra $\Delta I\left(\nu, \nu_{\text {opt }}\right)$ for

binding energies of $E_{\mathrm{B}}=60 \mathrm{meV}(\mathbf{b}), E_{\mathrm{B}}=240 \mathrm{meV}(\mathbf{d})$ and $E_{\mathrm{B}}=600 \mathrm{meV}(\mathbf{f})$ and detunings $\Delta_{\text {opt }}$ as in the experiment shown in a. c, e, Measured (c) and calculated (e) HSG intensities (colour scale) for $\Delta_{\text {opt }}=0$ as functions of $\nu_{\mathrm{HSG}}$ and delay $t_{\mathrm{ex}}$. 